\title{
Synchronization of Target Tracking Cascaded Leader-Follower Spacecraft Formation
}

\author{
Rune Schlanbusch and Per Johan Nicklasson \\ Department of Technology, Narvik University College, PB 385, AN-8505 Narvik \\ Norway
}

\section{Introduction}

In recent years, formation flying has become an increasingly popular subject of study. This is a new method of performing space operations, by replacing large and complex spacecraft with an array of simpler micro-spacecraft bringing out new possibilities and opportunities of cost reduction, redundancy and improved resolution aspects of onboard payload. One of the main challenges is the requirement of synchronization between spacecraft; robust and reliable control of relative position and attitude are necessary to make the spacecraft cooperate to gain the possible advantages made feasible by spacecraft formations. For fully autonomous spacecraft formations both path- and attitude-planning must be performed on-line which introduces challenges like collision avoidance and restrictions on pointing instruments towards required targets, with the lowest possible fuel expenditure. The system model is a key element to achieve a reliable and robust controller.

\subsection{Previous work}

The simplest Cartesian model of relative motion between two spacecraft is linear and known as the Hill (Hill, 1878) or Clohessy-Wiltshire (Clohessy \& Wiltshire, 1960) equations; a linear model based on assumptions of circular orbits, no orbital perturbations and small relative distance between spacecraft compared with the distance from the formation to the center of the Earth. As the visions for tighter spacecraft formations in highly elliptic orbits appeared, the need for more detailed models arose, especially regarding orbital perturbations. This resulted in nonlinear models as presented in e.g. (McInnes, 1995; Wang \& Hadaegh, 1996), and later in (Yan et al., 2000a) and (Kristiansen, 2008), derived for arbitrary orbital eccentricity and with added terms for orbital perturbations. Most previous work on reference generation are concerned with translational trajectory generation for fuel optimal reconfiguration and formation keeping such as in (Wong \& Kapila, 2005) where a formation located at the Sun-Earth $L_{2}$ Langrange Point is considered, while (Yan et al., 2009) proposed two approaches to design perturbed satellite formation relative motion orbits using least-square techniques. Trajectory optimization for satellite reconfiguration maneuvers coupled with attitude constraints have been investigated in (Garcia \& How, 2005) where a path planner based on rapidly-exploring random tree is used in addition to a smoother function. Coupling between the position and attitude is introduced by the pointing constrains, and thus the trajectory design must be solved as a single $6 \mathrm{~N}$ Degrees of Freedom (DOF) problem instead of $N$ separate 6 DOF problems. 
Ground target tracking for spacecraft has been addressed by several other researchers, such as (Goerre \& Shucker, 1999; Chen et al., 2000; Tsiotras et al., 2001) and (Steyn, 2006) where only one spacecraft is considered. The usual way to generate target tracking reference is to find a vector pointing from the spacecraft towards a point on the planet surface where the instrument is supposed to be pointing, and then the desired quaternions and angular velocities are generated to ensure high accuracy tracking of the specified target point.

Due to the parameterization of the attitude for both Euler angles and the unit quaternion we obtain a set of two equilibria of the closed-loop system of a rigid body, and possibilities of the unwinding phenomenon. One approach to solve the problem of multiple equilibria is the use of hybrid control (cf. (Liberzon, 2003), (Goebel et al., 2009)), and different solutions have been presented, as in (Casagrande, 2008) for an underactuated non-symmetric rigid body, and by (Mayhew et al., 2009) using quaternion-based hybrid feedback where the choice of rotational direction is performed by a switching control law.

The nonlinear nature of the tracking control problem has been a challenging task in robotics and control research. The so called passivity-based approach to robot control have gained much attention, which, contrary to computed torque control, coupe with the robot control problem by exploiting the robots' physical structure (Berghuis \& Nijmeijer, 1993). A simple solution to the closed-loop passivity approach was proposed by (Takegaki \& Arimoto, 1981) on the robot position control problem. The natural extension the motion control task was solved in (Paden \& Panja, 1988), where the controller was called PD+, and in (Slotine \& Li, 1987) where the controller was called passivity-based sliding surface. The control structure was later applied for spacecraft formation control in (Kristiansen, 2008).

For large systems, e.g. complex dynamical systems such as spacecraft formations, the expression divide and conquer may seem appealing, and for good reasons; by dividing a system into smaller parts, the difficulties of stability analysis and control design can be greatly reduced. A particular case of such systems is cascaded structure which consists of a driving system which is an input to the driven system through an interconnection (see (Loría \& Panteley, 2005) and references therein).

The topic of cascaded systems have received a great deal of attention and has successfully been applied to a wide number of applications. In (Fossen \& Fjellstad, 1993) a cascaded adaptive control scheme for marine vehicles including actuator dynamics was introduced, while (Loría et al., 1998) solved the problem of synchronization of two pendula through use of cascades. The authors of (Janković et al., 1996) studied the problem of global stabilisability of feedforward systems by a systematic recursive design procedure for autonomous systems, while time-varying systems were considered in (Jiang \& Mareels, 1997) for stabilization of robust control, while (Panteley \& Loría, 1998) established sufficient conditions for Uniform Global Asymptotical Stability (UGAS) for cascaded nonlinear time-varying systems. The aspects of practical and semi-global stability for nonlinear time-varying systems in cascade were pursued in (Chaillet, 2006) and (Chaillet \& Loría, 2008). A stability analysis of spacecraft formations including both leader and follower using relative coordinates was presented in (Grøtli, 2010), where the controller-observer scheme was proven input-to-state-stable.

\subsection{Contribution}

In this paper we present a solution for real-time generation of attitude references for a leader-follower spacecraft formation with target tracking leader and followers complementing the measurement by pointing their instruments at a common target on the Earth surface. The solution is based on a 6DF model where each follower generates the 
attitude references in real-time based on relative translational motion between the leader and its followers, which also ensures that the spacecraft are pointing at the target during formation reconfiguration. We are utilizing a passivity-based sliding surface controller for relative position and Uniform Global Practical Asymptotic Stability (UGPAS) is proven for the equilibrium point of the closed-loop system. The control law is also adapted for hybrid switching control with hysteresis for attitude tracking spacecraft in formation to ensure robust stability when measurement noise is considered, and avoid unwinding, thus achieving Uniform Practical Asymptotical Stability (UPAS) in the large on the set $\mathcal{S}^{3} \times \mathbb{R}^{3}$ for the equilibrium point of the closed-loop system. Simulation results are presented to show how the attitude references are generated during a formation reconfiguration using the derived control laws.

The rest of the paper is organized as follows. In Section 2 we describe the modeling of relative translation and rotation for spacecraft formations; in Section 3 we present a scheme were the attitude reference for the leader and follower spacecraft is generated based on relative coordinates; in Section 4 we present continuous control of relative translation and hybrid control of relative rotation where stability of the overall system is proved through use of cascades; in Section 5 we present simulation results and we conclude with some remarks in Section 6.

\section{Modeling}

In the following, we denote by $\dot{\mathbf{x}}$ the time derivative of a vector $\mathbf{x}$, i.e. $\dot{\mathbf{x}}=d \mathbf{x} / d t$, and moreover, $\ddot{\mathbf{x}}=d^{2} \mathbf{x} / d t^{2}$. We denote by $\|\cdot\|$ the Euclidian norm of a vector and the induced $\mathcal{L}_{2}$ norm of a matrix. The cross-product operator is denoted $\mathbf{S}(\cdot)$, such that $\mathbf{S}(\mathbf{x}) \mathbf{y}=\mathbf{x} \times \mathbf{y}$. Reference frames are denoted by $\mathcal{F}^{(\cdot)}$, and in particular, the standard Earth-Centered Inertial (ECI) frame is denoted $\mathcal{F}^{i}$ and The Earth-Centered Earth-Fixed (ECEF) frame is denoted $\mathcal{F}^{e}$. We denote by $\boldsymbol{\omega}_{b, a}^{c}$ the angular velocity of frame $\mathcal{F}^{a}$ relative to frame $\mathcal{F}^{b}$, referenced in frame $\mathcal{F}^{c}$. Matrices representing rotation or coordinate transformation from frame $\mathcal{F}^{a}$ to frame $\mathcal{F}^{b}$ are denoted $\mathbf{R}_{a}^{b}$. When the context is sufficiently explicit, we may omit to write arguments of a function, vector or matrix.

\subsection{Cartesian coordinate frames}

Basically there are two different approaches for modeling spacecraft formations: Cartesian coordinates and orbital elements, which both have their pros and cons. The orbital element method is often used to design formations concerning low fuel expenditure because of the relationship towards natural orbits, while Cartesian models often are used where an orbit with fixed dimensions are studied, which is the case in this paper.

The coordinate reference frames used throughout the paper are shown in Figure 1, and defined as follows:

Leader orbit reference frame: The leader orbit frame, denoted $\mathcal{F}^{l}$, has its origin located in the center of mass of the leader spacecraft. The $\mathbf{e}_{r}$ axis in the frame coincide with the vector $\mathbf{r}_{l} \in \mathbb{R}^{3}$ from the center of the Earth to the spacecraft, and the $\mathbf{e}_{h}$ axis is parallel to the orbital angular momentum vector, pointing in the orbit normal direction. The $\mathbf{e}_{\theta}$ axis completes the right-handed orthonormal frame. The basis vectors of the frame can be defined as

$$
\mathbf{e}_{r}:=\frac{\mathbf{r}_{l}}{\left\|\mathbf{r}_{l}\right\|}, \quad \mathbf{e}_{\theta}:=\mathbf{S}\left(\mathbf{e}_{h}\right) \mathbf{e}_{r} \quad \text { and } \quad \mathbf{e}_{h}:=\frac{\mathbf{h}}{\|\mathbf{h}\|},
$$

where $\mathbf{h}=\mathbf{S}\left(\mathbf{r}_{l}\right) \dot{\mathbf{r}}_{l}$ is the angular momentum vector of the orbit. 
Follower orbit reference frame: The follower orbit frame has its origin in the center of mass of the follower spacecraft, and is denoted $\mathcal{F}^{f}$. The vector pointing from the center of the Earth to the frame origin is denoted $\mathbf{r}_{f} \in \mathbb{R}^{3}$, and the frame is specified by a relative orbit position vector $\mathbf{p}=[x, y, z]^{\top}$ expressed in $\mathcal{F}^{l}$ components, and its unit vectors align with the basis vectors of $\mathcal{F}^{l}$. Accordingly,

$$
\mathbf{p}=\mathbf{R}_{i}^{l}\left(\mathbf{r}_{f}-\mathbf{r}_{l}\right)=x \mathbf{e}_{r}+y \mathbf{e}_{\theta}+z \mathbf{e}_{h} \Rightarrow \mathbf{r}_{f}=\mathbf{R}_{l}^{i} \mathbf{p}+\mathbf{r}_{l}
$$

\subsection{Quaternions and kinematics}

The attitude of a rigid body is often represented by a rotation matrix $\mathbf{R} \in S O$ (3) fulfilling

$$
S O(3)=\left\{\mathbf{R} \in \mathbb{R}^{3 \times 3}: \mathbf{R}^{\top} \mathbf{R}=\mathbf{I}, \operatorname{det} \mathbf{R}=1\right\},
$$

which is the special orthogonal group of order three, where I denotes the identity matrix. A rotation matrix for a rotation $\theta$ about an arbitrary unit vector $k \in \mathbb{R}^{3}$ can be angle-axis parameterized as $-c f$. (Egeland \& Gravdahl, 2002),

$$
\mathbf{R}_{k, \theta}=\mathbf{I}+\mathbf{S}(\mathbf{k}) \sin \theta+\mathbf{S}^{2}(\mathbf{k})(1-\cos \theta),
$$

and coordinate transformation of a vector $\mathbf{r}$ from frame $a$ to frame $b$ is written as $\mathbf{r}^{b}=\mathbf{R}_{a}^{b} \mathbf{r}^{a}$. The rotation matrix in (4) can be expressed by an Euler parameter representation as

$$
\mathbf{R}=\mathbf{I}+2 \eta \mathbf{S}(\boldsymbol{\epsilon})+2 \mathbf{S}^{2}(\boldsymbol{\epsilon}),
$$

where the matrix $\mathbf{S}(\cdot)$ is the cross product operator

$$
\mathbf{S}(\boldsymbol{\epsilon})=\boldsymbol{\epsilon} \times=\left[\begin{array}{ccc}
0 & -\epsilon_{z} & \epsilon_{y} \\
\epsilon_{z} & 0 & -\epsilon_{x} \\
-\epsilon_{y} & \epsilon_{x} & 0
\end{array}\right], \boldsymbol{\epsilon}=\left[\begin{array}{c}
\epsilon_{x} \\
\epsilon_{y} \\
\epsilon_{z}
\end{array}\right]
$$

Quaternions are often used to parameterize members of $S O(3)$ where the unit quaternion is defined as $\mathbf{q}=\left[\eta, \boldsymbol{\epsilon}^{\top}\right]^{\top} \in \mathcal{S}^{3}=\left\{\mathbf{x} \in \mathbb{R}^{4}: \mathbf{x}^{\top} \mathbf{x}=1\right\}$, where $\eta=\cos (\theta / 2) \in \mathbb{R}$ is the scalar

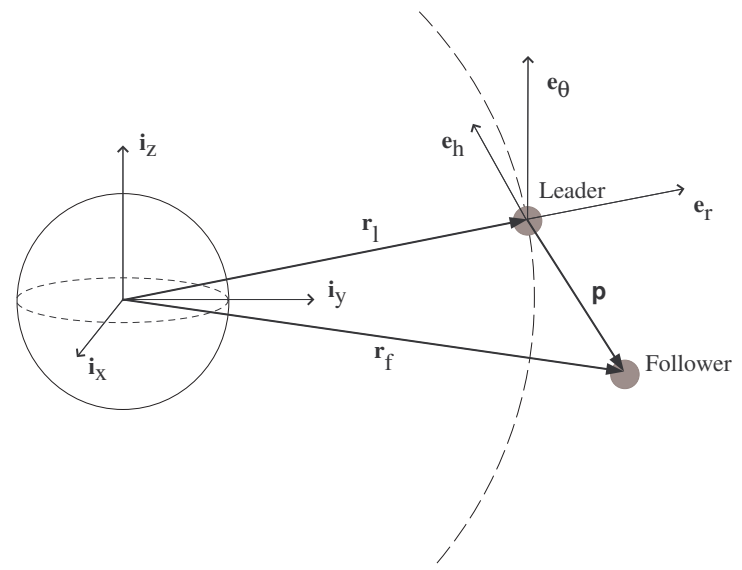

Fig. 1. Reference coordinate frames. 
part and $\boldsymbol{\epsilon}=\mathbf{k} \sin (\theta / 2) \in \mathbb{R}^{3}$ is the vector part. The set $\mathcal{S}^{3}$ forms a group with quaternion multiplication, which is distributive and associative, but not commutative, and the quaternion product is defined as

$$
\mathbf{q}_{1} \otimes \mathbf{q}_{2}=\left[\begin{array}{c}
\eta_{1} \eta_{2}-\boldsymbol{\epsilon}_{1}^{\top} \boldsymbol{\epsilon}_{2} \\
\eta_{1} \boldsymbol{\epsilon}_{2}+\eta_{2} \boldsymbol{\epsilon}_{1}+\mathbf{S}\left(\boldsymbol{\epsilon}_{1}\right) \boldsymbol{\epsilon}_{2}
\end{array}\right]
$$

The inverse rotation can be performed by using the inverse conjugate of $\mathbf{q}$ given by $\overline{\mathbf{q}}=$ $\left[\eta,-\boldsymbol{\epsilon}^{\top}\right]^{\top}$. The time derivative of the rotation matrix is

$$
\dot{\mathbf{R}}_{b}^{a}=\mathbf{S}\left(\boldsymbol{\omega}_{a, b}^{a}\right) \mathbf{R}_{b}^{a}=\mathbf{R}_{b}^{a} \mathbf{S}\left(\boldsymbol{\omega}_{a, b}^{b}\right),
$$

and the kinematic differential equations may be expressed as

$$
\dot{\mathbf{q}}=\mathbf{T}(\mathbf{q}) \boldsymbol{\omega}, \mathbf{T}(\mathbf{q})=\frac{1}{2}\left[\begin{array}{c}
-\boldsymbol{\epsilon}^{T} \\
\eta \mathbf{I}+\mathbf{S}(\boldsymbol{\epsilon})
\end{array}\right] \in \mathbb{R}^{4 \times 3} .
$$

\subsubsection{Relative translation}

The fundamental differential equation of the two-body problem can be expressed as ( $c f$. (Battin, 1999))

$$
\ddot{\mathbf{r}}_{s}=-\frac{\mu}{r_{s}^{3}} \mathbf{r}_{s}+\frac{\mathbf{f}_{s d}}{m_{s}}+\frac{\mathbf{f}_{s a}}{m_{s}}
$$

where $\mathbf{f}_{s d} \in \mathbb{R}^{3}$ is the perturbation term due to external effects, $\mathbf{f}_{s a} \in \mathbb{R}^{3}$ is the actuator force, $m_{S}$ is the mass of the spacecraft, and super-/sub-script $s$ denotes the spacecraft in question, so $s=l, f$ for the leader and follower spacecraft respectively. The spacecraft masses are assumed to be small relative to the mass of the Earth $M_{e}$, so $\mu \approx G M_{e}$, where $G$ is the gravitational constant. According to (2) the relative position between the leader and follower spacecraft may be expressed as

$$
\mathbf{R}_{l}^{i} \mathbf{p}=\mathbf{r}_{f}-\mathbf{r}_{l},
$$

and by differentiating twice we obtain

$$
\mathbf{R}_{l}^{i} \ddot{\mathbf{p}}+2 \mathbf{R}_{l}^{i} \mathbf{S}\left(\boldsymbol{\omega}_{i, l}^{l}\right) \dot{\mathbf{p}}+\mathbf{R}_{l}^{i}\left(\mathbf{S}^{2}\left(\boldsymbol{\omega}_{i, l}^{l}\right)+\mathbf{S}\left(\dot{\boldsymbol{\omega}}_{i, l}^{l}\right)\right) \mathbf{p}=\ddot{\mathbf{r}}_{f}-\ddot{\mathbf{r}}_{l} .
$$

By inserting (10), the right hand side of (12) may be written as

$$
\ddot{\mathbf{r}}_{f}-\ddot{\mathbf{r}}_{l}=-\frac{\mu}{r_{f}^{3}} \mathbf{r}_{f}+\frac{\mathbf{f}_{f d}}{m_{f}}+\frac{\mathbf{f}_{f a}}{m_{f}}+\frac{\mu}{r_{l}^{3}} \mathbf{r}_{l}-\frac{\mathbf{f}_{l d}}{m_{l}}-\frac{\mathbf{f}_{l a}}{m_{l}},
$$

and by inserting (2) into (13), we find that

$$
m_{f}\left(\ddot{\mathbf{r}}_{f}-\ddot{\mathbf{r}}_{l}\right)=-m_{f} \mu\left[\left(\frac{1}{r_{f}^{3}}-\frac{1}{r_{l}^{3}}\right) \mathbf{r}_{1}+\frac{\mathbf{R}_{l}^{i} \mathbf{p}}{r_{f}^{3}}\right]+\mathbf{f}_{f a}+\mathbf{f}_{f d}-\frac{m_{f}}{m_{l}}\left(\mathbf{f}_{l a}+\mathbf{f}_{l d}\right) .
$$

Moreover, by inserting (14) into (12), and rearranging the terms we obtain

$$
m_{f} \ddot{\mathbf{p}}+\mathbf{C}_{t}\left(\boldsymbol{\omega}_{i, l}^{l}\right) \dot{\mathbf{p}}+\mathbf{D}_{t}\left(\dot{\boldsymbol{\omega}}_{i, l}^{l}, \boldsymbol{\omega}_{i, l}^{l}, r_{f}\right) \mathbf{p}+\mathbf{n}_{t}\left(\mathbf{r}_{l}, r_{f}\right)=\mathbf{F}_{a}+\mathbf{F}_{d},
$$


where

$$
\mathbf{C}_{t}\left(\boldsymbol{\omega}_{i, l}^{l}\right)=2 m_{f} \mathbf{S}\left(\boldsymbol{\omega}_{i, l}^{l}\right)
$$

is a skew-symmetric matrix,

$$
\mathbf{D}_{t}\left(\dot{\boldsymbol{\omega}}_{i, l}^{l}, \boldsymbol{\omega}_{i, l}^{l}, r_{f}\right)=m_{f}\left[\mathbf{S}^{2}\left(\boldsymbol{\omega}_{i, l}^{l}\right)+\mathbf{S}\left(\dot{\boldsymbol{\omega}}_{i, l}^{l}\right)+\frac{\mu}{r_{f}^{3}} \mathbf{I}\right]
$$

may be viewed as a time-varying potential force, and

$$
\mathbf{n}_{t}\left(\mathbf{r}_{l}, r_{f}\right)=\mu m_{f} \mathbf{R}_{i}^{l}\left[\frac{1}{r_{f}^{3}}-\frac{1}{r_{l}^{3}}\right] \mathbf{r}_{l}
$$

is a nonlinear term. The composite perturbation force $\mathbf{F}_{d}$ and the composite relative control force $\mathbf{F}_{a}$ are respectively written as

$$
\mathbf{F}_{d}=\mathbf{R}_{i}^{l}\left(\mathbf{f}_{f d}-\frac{m_{f}}{m_{l}} \mathbf{f}_{l d}\right) \quad \text { and } \quad \mathbf{F}_{a}=\mathbf{R}_{i}^{l}\left(\mathbf{f}_{f a}-\frac{m_{f}}{m_{l}} \mathbf{f}_{l a}\right) .
$$

Note that all forces $\mathbf{f}$ are presented in the inertial frame. If the forces are computed in another frame, the rotation matrix should be replaced accordingly. The orbital angular velocity and angular acceleration can be expressed as $\omega_{i, l}^{i}=\mathbf{S}\left(\mathbf{r}_{l}\right) \mathbf{v}_{l} / \mathbf{r}_{l}^{\top} \mathbf{r}_{l}$, and

$$
\dot{\omega}_{i, l}^{i}=\frac{\mathbf{r}_{l}^{\top} \mathbf{r}_{l} \mathbf{S}\left(\mathbf{r}_{l}\right) \mathbf{a}_{l}-2 \mathbf{v}_{l}^{\top} \mathbf{r}_{l} \mathbf{S}\left(\mathbf{r}_{l}^{\top}\right) \mathbf{v}_{l}}{\left(\mathbf{r}_{l}^{\top} \mathbf{r}_{l}\right)^{2}},
$$

respectively.

\subsubsection{Relative rotation}

With the assumptions of rigid body movement, the dynamical model of a spacecraft can be found from Euler's momentum equations as (Sidi, 1997)

$$
\begin{aligned}
\mathbf{J}_{s} \dot{\boldsymbol{\omega}}_{i, s b}^{s b} & =-\mathbf{S}\left(\boldsymbol{\omega}_{i, s b}^{s b}\right) \mathbf{J}_{s} \boldsymbol{\omega}_{i, s b}^{s b}+\boldsymbol{\tau}_{s d}^{s b}+\boldsymbol{\tau}_{s a}^{s b} \\
\boldsymbol{\omega}_{s, s b}^{s b} & =\boldsymbol{\omega}_{i, s b}^{s b}-\mathbf{R}_{i}^{s b} \boldsymbol{\omega}_{i, s^{\prime}}^{i}
\end{aligned}
$$

where $\mathbf{J}_{s}=\operatorname{diag}\left\{J_{s x}, J_{s y}, J_{s z}\right\} \in \mathbb{R}^{3 \times 3}$ is the spacecraft moment of inertia matrix, $\boldsymbol{\tau}_{s d}^{s b} \in \mathbb{R}^{3}$ is the total disturbance torque, $\boldsymbol{\tau}_{s a}^{s b} \in \mathbb{R}^{3}$ is the total actuator torque and $\boldsymbol{\omega}_{i, s}^{i}=\mathbf{S}\left(\mathbf{r}_{s}\right) \mathbf{v}_{s} / \mathbf{r}_{s}^{\top} \mathbf{r}_{s}$ is the orbital angular velocity. Rotation from the leader body frame to the inertial frame are denoted $\mathbf{q}_{l b}^{i}$, while rotation from the follower body frame to the inertial frame are denoted $\mathbf{q}_{f b}^{i}$. Relative rotation between the follower and leader body frame is found by applying the quaternion product (cf. (7)) expressed as

$$
\mathbf{q}_{f b}^{l b}=\mathbf{q}_{f b}^{i} \otimes \overline{\mathbf{q}}_{l b}^{i}
$$

and with a slightly abuse of notation we denote $\mathbf{q}_{l}=\mathbf{q}_{l b}^{i}$ and $\mathbf{q}_{f}=\mathbf{q}_{f b}^{l b}$. The relative attitude dynamics may be expressed as (cf. (Yan et al., 2000b; Kristiansen, 2008))

$$
\begin{aligned}
\mathbf{J}_{f} \dot{\boldsymbol{\omega}} & +\mathbf{J}_{f} \mathbf{S}\left(\mathbf{R}_{l b}^{f b} \boldsymbol{\omega}_{i, l b}^{l b}\right) \boldsymbol{\omega}-\mathbf{J}_{f} \mathbf{R}_{l b}^{f b} \mathbf{J}_{l}^{-1} \mathbf{S}\left(\boldsymbol{\omega}_{i, l b}^{l b}\right) \mathbf{J}_{l} \boldsymbol{\omega}_{i, l b}^{l b} \\
& +\mathbf{S}\left(\boldsymbol{\omega}+\mathbf{R}_{l b}^{f b} \boldsymbol{\omega}_{i, l b}^{l b}\right) \mathbf{J}_{f}\left(\boldsymbol{\omega}+\mathbf{R}_{l b}^{f b} \boldsymbol{\omega}_{i, l b}^{l b}\right)=\mathrm{Y}_{d}+\mathrm{Y}_{a}
\end{aligned}
$$


where

$$
\boldsymbol{\omega}=\boldsymbol{\omega}_{i, f b}^{f b}-\mathbf{R}_{l b}^{f b} \boldsymbol{\omega}_{i, l b}^{l b}
$$

is the relative angular velocity between the follower body reference frame and the leader body reference frame expressed in the follower body reference frame,

$$
\mathrm{Y}_{d}=\boldsymbol{\tau}_{f d}^{f b}-\mathbf{J}_{f} \mathbf{R}_{l b}^{f b} \mathbf{J}_{l}^{-1} \boldsymbol{\tau}_{l d}^{l b}, \mathrm{Y}_{a}=\boldsymbol{\tau}_{f a}^{f b}-\mathbf{J}_{f} \mathbf{R}_{l b}^{f b} \mathbf{J}_{l}^{-1} \boldsymbol{\tau}_{l a}^{l b}
$$

are the relative perturbation torque and actuator torque, respectively. For simplicity (24) may be rewritten as

$$
\mathbf{J}_{f} \dot{\boldsymbol{\omega}}+\mathbf{C}_{r}(\boldsymbol{\omega}) \boldsymbol{\omega}+\mathbf{n}_{r}(\boldsymbol{\omega})=\mathrm{Y}_{d}+\mathrm{Y}_{a},
$$

where

$$
\mathbf{C}_{r}(\boldsymbol{\omega})=\mathbf{J}_{f} \mathbf{S}\left(\mathbf{R}_{l b}^{f b} \boldsymbol{\omega}_{i, l b}^{l b}\right)+\mathbf{S}\left(\mathbf{R}_{l b}^{f b} \boldsymbol{\omega}_{i, l b}^{l b}\right) \mathbf{J}_{f}-\mathbf{S}\left(\mathbf{J}_{f}\left(\boldsymbol{\omega}+\mathbf{R}_{l b}^{f b} \boldsymbol{\omega}_{i, l b}^{l b}\right)\right)
$$

is a skew-symmetric matrix, and

$$
\mathbf{n}_{r}(\boldsymbol{\omega})=\mathbf{S}\left(\mathbf{R}_{l b}^{f b} \boldsymbol{\omega}_{i, l b}^{l b}\right) \mathbf{J}_{f} \mathbf{R}_{l b}^{f b} \boldsymbol{\omega}_{i, l b}^{l b}-\mathbf{J}_{f} \mathbf{R}_{l b}^{f b} \mathbf{J}_{l}^{-1} \mathbf{S}\left(\boldsymbol{\omega}_{i, l b}^{l b}\right) \mathbf{J}_{l} \boldsymbol{\omega}_{i, l b}^{l b}
$$

is a nonlinear term.

\section{Reference generation}

Our objective for the spacecraft formation is to have each spacecraft, including the leader, tracking a fixed point located at the surface of e.g. the Earth by specifying a tracking direction of the selected pointing axis where a measurement instrument is mounted such as e.g. a camera or antenna. The target is chosen by the spacecraft operator as a given set of coordinates such as latitude $(\phi)$ and longitude $(\lambda)$. The vector pointing from the center of Earth to the target in an Earth Centered Earth Fixed (ECEF) frame is obtained by applying

$$
\mathbf{r}_{t}^{e}=\left[\begin{array}{c}
\cos (\phi) \cos (\lambda) \\
\cos (\phi) \sin (\lambda) \\
\sin (\phi)
\end{array}\right] r_{e}
$$

where $r_{e}=6378.137 \times 10^{3} \mathrm{~m}$ is the Earth radii. It is assumed a perfect spherical Earth; alternatively a function of the Earth radii may be used as $r_{e}(\lambda, \phi)$ with longitude and latitude as arguments. If we assume that the Earth has a constant angular rate $\omega_{e}=7.292115 \times$ $10^{-5} \mathrm{rad} / \mathrm{s}$ around its rotation axis we can rotate the target vector to ECI coordinates by utilizing

$$
\mathbf{r}_{t}=\mathbf{R}_{e}^{i} \mathbf{r}_{t}^{e}
$$

where the rotation matrix from ECEF to ECI coordinates is dentoed

$$
\mathbf{R}_{e}^{i}=\left[\begin{array}{ccc}
\cos \left(\omega_{e} t+\alpha\right) & -\sin \left(\omega_{e} t+\alpha\right) & 0 \\
\sin \left(\omega_{e} t+\alpha\right) & \cos \left(\omega_{e} t+\alpha\right) & 0 \\
0 & 0 & 1
\end{array}\right],
$$

where $t$ is time scalar and $\alpha$ is an initial phase between the $x$-axis of the ECEF and EIC coordinates at $t=0$. 


\subsection{Leader reference}

For the leader spacecraft we start by defining a target pointing vector in inertial coordinates as

$$
\mathbf{1}_{l d}=\mathbf{r}_{t}-\mathbf{r}_{l},
$$

which is used to construct a leader desired frame called $\mathcal{F}^{l d}$ as

$$
\mathbf{x}_{l d}=-\frac{\mathbf{1}_{l d}}{\left\|\mathbf{1}_{l d}\right\|}, \quad \mathbf{y}_{l d}=\frac{\mathbf{S}\left(\mathbf{x}_{l d}\right)\left(-\mathbf{h}_{l}\right)}{\left\|\mathbf{S}\left(\mathbf{x}_{l d}\right)\left(-\mathbf{h}_{l}\right)\right\|} \quad \text { and } \quad \mathbf{z}_{l d}=\mathbf{S}\left(\mathbf{x}_{l d}\right) \mathbf{y}_{l d},
$$

and thus we can obtain a desired quaternion vector by transforming the constructed rotation matrix and require continuity of solution to ensure a smooth vector over time. By differentiating (33) twice we obtain

$$
\begin{aligned}
& \dot{\mathrm{i}}_{l d}=\dot{\mathbf{r}}_{t}-\dot{\mathbf{r}}_{i}, \\
& \ddot{\mathrm{i}}_{l d}=\ddot{\mathbf{r}}_{t}-\ddot{\mathbf{r}}_{i},
\end{aligned}
$$

where

$$
\begin{aligned}
& \dot{\mathbf{r}}_{t}=\mathbf{S}\left(\boldsymbol{\omega}_{i, e}^{i}\right) \mathbf{R}_{e}^{i} \mathbf{r}_{t}^{e}, \\
& \ddot{\mathbf{r}}_{t}=\mathbf{S}^{2}\left(\boldsymbol{\omega}_{i, e}^{i}\right) \mathbf{R}_{e}^{i} \mathbf{r}_{t}^{e},
\end{aligned}
$$

and $\boldsymbol{\omega}_{i, e}^{i}=\left[0,0, \omega_{e}\right]^{\top}$. According to $($ Wertz, 1978) the relationship between the desired angular velocity and the normalized target vector is

$$
\dot{\ell}_{l d}=\mathbf{S}\left(\boldsymbol{\omega}_{i, l d}^{i}\right) \boldsymbol{\ell}_{l d}
$$

where

$$
\ell_{l d}=\mathbf{1}_{l d} /\left\|\mathbf{1}_{l d}\right\| \text {. }
$$

Equ. (39) is linearly dependent, thus the desired angular velocity is not uniquely specified. On component form (39) is written as

$$
\begin{aligned}
& \dot{\ell}_{l d x}=-\omega_{l d z} \ell_{l d y}+\omega_{l d y} \ell_{l d z}, \\
& \dot{\ell}_{l d y}=\omega_{l d z} \ell_{l d x}-\omega_{l d x} \ell_{l d z}, \\
& \dot{\ell}_{l d z}=-\omega_{l d y} \ell_{l d x}+\omega_{l d x} \ell_{l d y},
\end{aligned}
$$

where $\boldsymbol{\omega}_{i, l d}^{i}=\left[\omega_{l d x}, \omega_{l d y}, \omega_{l d z}\right]^{\top}$ and $\ell_{l d}=\left[\ell_{l d x}, \ell_{l d y}, \ell_{l d z}\right]^{\top}$. This particular problem was solved in (Chen et al., 2000) by adding a cost constraint to minimize the amplitude of $\boldsymbol{\omega}_{i, l d}^{i}$ such as

$$
J=\frac{1}{2} k \boldsymbol{\omega}_{i, l d}^{i, \top} \boldsymbol{\omega}_{i, l d}^{i}
$$

where $k$ is a positive cost scalar. We then define a Hamiltonian function based on (41b) and (41c) leading to

$$
\mathcal{H}=\frac{1}{2} k \boldsymbol{\omega}_{i, l d}^{i, T} \boldsymbol{\omega}_{i, l d}^{i}+\lambda_{1}\left(\dot{\ell}_{l d y}-\omega_{l d z} \ell_{l d x}+\omega_{l d x} l_{l d z}\right)+\lambda_{2}\left(\dot{\ell}_{l d z} \omega_{l d y} \ell_{l d x}-\omega_{l d x} \ell_{l d y}\right),
$$


where $\lambda_{1}, \lambda_{2}$ are constant adjoint scalars. By differentiating (43) with respect to $\omega_{i, l d}^{i}$ and setting the result to zero, we obtain

$$
\begin{aligned}
& k \omega_{l d x}+\lambda_{1} \ell_{l d z}-\lambda_{2} \ell_{l d y}=0, \\
& k \omega_{l d y}+\lambda_{2} \ell_{l d x}=0, \\
& k \omega_{l d z}-\lambda_{1} \ell_{l d x}=0 .
\end{aligned}
$$

By inserting (44b) and (44c) into (44a) we obtain the relation

$$
\omega_{i, l d}^{i} \cdot \ell_{l d}=0,
$$

which implies that the desired angular velocity will be orthogonal to the desired tracking direction. By solving (39) and (45) for the angular velocity, we obtain

$$
\boldsymbol{\omega}_{i, l d}^{i}=\mathbf{S}\left(\ell_{l d}\right) \dot{\ell}_{l d},
$$

which is a solution resulting in no rotation about the desired pointing direction during tracking maneuvers. By inserting (40) and its differentiated into (46), it can be shown that

$$
\boldsymbol{\omega}_{i, l d}^{i}=\frac{\mathbf{S}\left(\mathbf{1}_{l d}\right) \dot{\mathbf{l}}_{l d}}{\left\|\mathbf{1}_{l d}\right\|^{2}}
$$

To obtain the desired angular acceleration we differentiate (47), which leads to the expression

$$
\dot{\omega}_{i, l d}^{i}=\frac{\mathbf{S}\left(\mathbf{l}_{l d}\right) \ddot{\mathbf{l}}_{l d}\left\|\mathbf{1}_{l d}\right\|^{2}-2 \mathbf{l}_{l d}^{\top} \mathbf{S}\left(\mathbf{l}_{l d}\right) \dot{\mathbf{l}}_{l d}}{\left\|\mathbf{1}_{l d}\right\|^{2}}
$$

Since the leader body frame is utilized in the dynamic equations (21), we simply rotate (47) and (48), obtaining

$$
\begin{aligned}
& \boldsymbol{\omega}_{i, l d}^{l b}=\mathbf{R}_{i}^{l b} \boldsymbol{\omega}_{i, l d}^{i}, \\
& \dot{\boldsymbol{\omega}}_{i, l d}^{l b}=-\mathbf{S}\left(\boldsymbol{\omega}_{i, l b}^{l b}\right) \mathbf{R}_{i}^{l b} \boldsymbol{\omega}_{i, l d}^{i}+\mathbf{R}_{i}^{l b} \dot{\boldsymbol{\omega}}_{i, l d}^{i} .
\end{aligned}
$$

\subsection{Follower reference}

The procedure to generate a follower reference is similar to the one presented in Section 3.1. We start by defining a target pointing vector in the inertial frame as

$$
\mathbf{1}_{f d}=\mathbf{r}_{t}-\mathbf{r}_{l}-\mathbf{R}_{l o}^{i} \mathbf{p},
$$

which is used to construct a follower desired reference frame called $\mathcal{F}^{f d}$ as

$$
\mathbf{x}_{f d}=-\frac{\mathbf{1}_{f d}}{\left\|\mathbf{1}_{f d}\right\|}, \quad \mathbf{y}_{f d}=\frac{\mathbf{S}\left(\mathbf{x}_{f d}\right)\left(-\mathbf{h}_{l}\right)}{\left\|\mathbf{S}\left(\mathbf{x}_{f d}\right)\left(-\mathbf{h}_{l}\right)\right\|} \quad \text { and } \quad \mathbf{z}_{f d}=\mathbf{S}\left(\mathbf{x}_{f d}\right) \mathbf{y}_{f d} .
$$

We can now construct a rotation matrix between $\mathcal{F}^{f d}$ and $\mathcal{F}^{i}$, and because the relative rotation is between $\mathcal{F}^{f b}$ and $\mathcal{F}^{l b}$ we apply composite rotation, thus obtaining

$$
\mathbf{R}_{f d}^{l b}=\mathbf{R}_{i}^{l b} \mathbf{R}_{f d}^{i}
$$


and transform the rotation matrix (53) to desired quaternion. By differentiating (51) twice, we obtain

$$
\begin{aligned}
& \dot{\mathbf{i}}_{f d}=\dot{\mathbf{r}}_{t}-\dot{\mathbf{r}}_{l}-\mathbf{S}\left(\boldsymbol{\omega}_{i, l o}^{i}\right) \mathbf{R}_{l o}^{i} \mathbf{p}-\mathbf{R}_{l o}^{i} \dot{\mathbf{p}}, \\
& \ddot{\mathbf{i}}_{f d}=\ddot{\mathbf{r}}_{t}-\ddot{\mathbf{r}}_{l}-\left(\mathbf{S}\left(\dot{\boldsymbol{\omega}}_{i, l o}^{i}\right)+\mathbf{S}^{2}\left(\boldsymbol{\omega}_{i, l o}^{i}\right)\right) \mathbf{R}_{l o}^{i} \mathbf{p}-2 \mathbf{S}\left(\boldsymbol{\omega}_{i, l o}^{i}\right) \mathbf{R}_{l o}^{i} \dot{\mathbf{p}}-\mathbf{R}_{l o}^{i} \ddot{\mathbf{p}} .
\end{aligned}
$$

The same optimization technique as presented in Section 3.1 can then be applied, leading to

$$
\begin{aligned}
\boldsymbol{\omega}_{i, f d}^{i} & =\frac{\mathbf{S}\left(\mathbf{1}_{f d}\right) \dot{\mathbf{1}}_{f d}}{\left\|\mathbf{1}_{f d}\right\|^{2}}, \\
\dot{\omega}_{i, f d}^{i} & =\frac{\mathbf{S}\left(\mathbf{1}_{f d}\right) \ddot{\mathbf{l}}_{f d}\left\|\mathbf{1}_{f d}\right\|^{2}-2 \mathbf{l}_{f d}^{\top} \mathbf{S}\left(\mathbf{1}_{f d}\right) \dot{\mathbf{1}}_{f d}}{\left\|\mathbf{1}_{f d}\right\|^{2}} .
\end{aligned}
$$

The desired angular rotation and acceleration vectors have to be transformed according to the relative dynamics of (24), resulting in

$$
\begin{aligned}
& \boldsymbol{\omega}_{l b, f d}^{f b}=\mathbf{R}_{i}^{f b} \boldsymbol{\omega}_{i, f d}^{i}-\mathbf{R}_{l b}^{f b} \boldsymbol{\omega}_{i, l b}^{l b}, \\
& \dot{\boldsymbol{\omega}}_{l b, f d}^{f b}=-\mathbf{S}\left(\boldsymbol{\omega}_{i, f b}^{f b}\right) \mathbf{R}_{i}^{f b} \boldsymbol{\omega}_{i, f d}^{i}+\mathbf{R}_{i}^{f b} \dot{\boldsymbol{\omega}}_{i, f d}^{i}+\mathbf{S}\left(\boldsymbol{\omega}_{l b, f b}^{f b}\right) \mathbf{R}_{l b}^{f b} \boldsymbol{\omega}_{i, l b}^{l b}-\mathbf{R}_{l b}^{f b} \dot{\boldsymbol{\omega}}_{i, l b}^{l b} .
\end{aligned}
$$

\section{Controller design}

In this section we present a control law for relative translation and switching control laws for attitude control of the leader spacecraft and relative attitude control for the follower spacecraft. All controllers are reminiscent of the so-called Slotine and Li controller for robot manipulators $-c f$. (Slotine \& Li, 1987). For all control laws it is assumed that all disturbances are unknown but upper bounded.

\subsection{Translational control}

We assume that disturbances for both the leader and follower spacecraft are unknown but bounded such that $\left\|\mathbf{f}_{l d}\right\| \leq \alpha_{l d}$ and $\left\|\mathbf{f}_{f d}\right\| \leq \alpha_{f d}$. In addition we also assume that control force of the leader spacecraft is upper bounded such that $\left\|\mathbf{f}_{l a}\right\| \leq \alpha_{l a}$. Reference trajectories are defined as

$$
\dot{\mathbf{p}}_{r}=\dot{\mathbf{p}}_{d}-\gamma \tilde{\mathbf{p}}, \quad \ddot{\mathbf{p}}_{r}=\ddot{\mathbf{p}}_{d}-\gamma \dot{\tilde{\mathbf{p}}},
$$

where $\mathbf{p}_{d}$ is the desired position, $\gamma>0$ is a constant gain, and $\tilde{\mathbf{p}}=\mathbf{p}-\mathbf{p}_{d}$ is the position error. The reference vector $\dot{\mathbf{p}}_{r}$ represent a notational manipulation that allows translation of energy-related properties expressed in terms of the actual velocity vector $\dot{\mathbf{p}}$ into trajectory control properties expressed in terms of the virtual velocity error vector $\mathbf{s}$. This is performed by shifting the desired velocities $\dot{\mathbf{p}}_{d}$ according to the position error $\tilde{\mathbf{p}}$ ( $c f$. (Slotine \& Li, 1987; Berghuis \& Nijmeijer, 1993)). The sliding surface is defined as

$$
\mathbf{s}=\dot{\mathbf{p}}-\dot{\mathbf{p}}_{r}=\dot{\tilde{\mathbf{p}}}+\gamma \tilde{\mathbf{p}} .
$$

A model based control law is derived based on (15) as

$$
\mathbf{f}_{f a}=m_{f} \ddot{\mathbf{p}}_{r}+\mathbf{C}_{t}\left(\boldsymbol{\omega}_{i, l}^{l}\right) \dot{\mathbf{p}}_{r}+\mathbf{D}_{t}\left(\dot{\boldsymbol{\omega}}_{i, l}^{l}, \boldsymbol{\omega}_{i, l}^{l}, r_{f}\right) \mathbf{p}+\mathbf{n}_{t}\left(\mathbf{r}_{l}, r_{f}\right)-\mathbf{K}_{p} \tilde{\mathbf{p}}-\mathbf{K}_{d} \mathbf{s}
$$


where $\mathbf{K}_{p}$ and $\mathbf{K}_{d}$ are both symmetric positive definite constant matrices such that $\mathbf{K}_{p}=\mathbf{K}_{p}^{\top}>$ 0 and $\mathbf{K}_{d}=\mathbf{K}_{d}^{\top}>0$. By inserting (62) and (61) into (15), the closed-loop dynamics may be written as

$$
m_{f} \dot{\mathbf{s}}+\left(\mathbf{C}_{t}+\mathbf{K}_{d}\right) \mathbf{s}+\mathbf{K}_{p} \tilde{\mathbf{p}}-\mathbf{f}_{f d}+\frac{m_{f}}{m_{l}}\left(\mathbf{f}_{l d}+\mathbf{f}_{l a}\right)=\mathbf{0} .
$$

A suitable Lypaunov Function Candidate (LFC) is chosen as

$$
V_{t}=\frac{1}{2} \mathbf{s}^{\top} m_{f} \mathbf{s}+\frac{1}{2} \tilde{\mathbf{p}}^{\top} \mathbf{K}_{p} \tilde{\mathbf{p}}>\mathbf{0} \quad \forall \mathbf{s} \neq \mathbf{0}, \tilde{\mathbf{p}} \neq \mathbf{0},
$$

and by differentiation and insertion of (63), we obtain

$$
\dot{V}_{t}=-\mathbf{s}^{\top} \mathbf{C}_{t} \mathbf{s}-\mathbf{s}^{\top} \mathbf{K}_{p} \tilde{\mathbf{p}}-\mathbf{s}^{\top} \mathbf{K}_{d} \mathbf{s}+\tilde{\mathbf{p}}^{\top} \mathbf{K}_{p} \dot{\tilde{\mathbf{p}}}+\mathbf{s}^{\top}\left(\mathbf{f}_{f d}-\frac{m_{f}}{m_{l}}\left(\mathbf{f}_{l d}+\mathbf{f}_{l a}\right)\right) .
$$

Using the fact that $\mathbf{C}_{t}\left(\boldsymbol{\omega}_{i, l}^{l}\right)$ is skew-symmetric, we further obtain

$$
\begin{aligned}
\dot{V}_{t} & =-\left(\mathbf{s}^{\top}-\dot{\tilde{\mathbf{p}}}^{\top}\right) \mathbf{K}_{p} \tilde{\mathbf{p}}-\mathbf{s}^{\top} \mathbf{K}_{d} \mathbf{s}+\mathbf{s}^{\top}\left(\mathbf{f}_{f d}-\frac{m_{f}}{m_{l}}\left(\mathbf{f}_{l d}+\mathbf{f}_{l a}\right)\right) \\
& =-\mathbf{x}_{t}^{\top} \mathbf{P} \mathbf{x}_{t}+\mathbf{s}^{\top}\left(\mathbf{f}_{f d}-\frac{m_{f}}{m_{l}}\left(\mathbf{f}_{l d}+\mathbf{f}_{l a}\right)\right) \\
& \leq-p_{m}\left\|\mathbf{x}_{t}\right\|^{2}+\left(\alpha_{f d}+\frac{m_{f}}{m_{l}}\left(\alpha_{l d}+\alpha_{l a}\right)\right)\left\|\mathbf{x}_{t}\right\|
\end{aligned}
$$

where $\mathbf{x}_{t}=\left[\mathbf{s}^{\top}, \tilde{\mathbf{p}}^{\top}\right]^{\top}, \mathbf{P}=\operatorname{diag}\left\{\mathbf{K}_{d}, \gamma \mathbf{K}_{p}\right\}$ and $p_{m}>0$ is the smallest eigenvalue of $\mathbf{P}$. Thus $\dot{V}_{t}<0$ when $\left\|\mathbf{x}_{t}\right\|>\delta_{t}=\left[\alpha_{f d}+\left(\alpha_{l d}+\alpha_{l a} m_{f} / m_{l}\right)\right] / p_{m}$ and $\delta_{t}$ can be diminished by increasing $p_{m}$ which is done by increasing the controller gains, and we can conclude that equilibrium point of the closed-loop system is Uniformly Globally Practically Exponentially Stable (UGPES) (cf. (Grøtli, 2010)).

Remark 1. If some of the unknown forces are assumed to be known, they can be removed by the control law thus putting less constraint on the controller gains.

Remark 2. We would also like to remark that even if we state global stability, this is not precise since when $\mathbf{R}_{l}^{i} \mathbf{p}=-\mathbf{r}_{l}$, which means that the follower is located at the center of the orbit, there is a singularity in (15) and according to (Hahn, 1967) the adjective global pertains to the case and only to the case when the state space is $\mathbb{R}^{n}$.

\subsection{Rotational control}

For attitude control, the system's solutions are defined using Teel's framework ( $c f$. (Goebel et al., 2009)) for hybrid systems and incorporates a switching law with hysteresis to coupe with the well known problem of dual equilibrium points when working with quaternion attitude representation. We assume that disturbances for both the leader and follower spacecraft are unknown but bounded such that $\left\|\boldsymbol{\tau}_{l d}^{l b}\right\| \leq \beta_{l d}$ and $\left\|\boldsymbol{\tau}_{f d}\right\| \leq \beta_{f d}$. The leader-follower dynamical system is looked upon as a cascaded system on the form

$$
\begin{aligned}
& \Sigma_{1}: \dot{x}_{1}=f_{1}\left(t, x_{1}\right)+g(t, x) \\
& \Sigma_{2}: \dot{x}_{2}=f_{2}\left(t, x_{2}\right),
\end{aligned}
$$


where $x_{1} \in \mathbb{R}^{n}, x_{2} \in \mathbb{R}^{m}, x=\left[x_{1}^{\top}, x_{2}^{\top}\right]^{\top}$ and the functions $f_{1}(\cdot, \cdot), f_{2}(\cdot, \cdot)$ and $g(\cdot, \cdot)$ are continuous in their arguments, locally Lipschitz in $x$, uniformly in $t$, and $f_{1}(\cdot, \cdot)$ is continuously differentiable in both arguments. Equation (70) is said to be the driving system,

$$
\dot{x}_{1}=f_{1}\left(t, x_{1}\right)
$$

is said to be the driven system, while the interconnection is the term $g(t, x)$. In our case the leader spacecraft is the driving system while the follower spacecraft represents the driven system.

The spacecraft error quaternion $\tilde{\mathbf{q}}_{s}=\left[\tilde{\eta}_{s}, \tilde{\boldsymbol{\epsilon}}_{s}^{\top}\right]^{\top}$ is given by the quaternion product (cf. (7))

$$
\tilde{\mathbf{q}}_{s}=\mathbf{q}_{s} \otimes \overline{\mathbf{q}}_{s d}=\left[\begin{array}{c}
\eta_{s} \eta_{s d}+\boldsymbol{\epsilon}_{s}^{\top} \boldsymbol{\epsilon}_{s d}^{\top} \\
\eta_{s d} \boldsymbol{\epsilon}_{s}-\eta_{s} \boldsymbol{\epsilon}_{s d}-\mathbf{S}\left(\boldsymbol{\epsilon}_{s}\right) \boldsymbol{\epsilon}_{s d}
\end{array}\right],
$$

and the error kinematics may be presented analogous to (9) as

$$
\dot{\tilde{\mathbf{q}}}_{s}=\mathbf{T}_{s e}\left(\tilde{\mathbf{q}}_{s}\right) \mathbf{e}_{s \omega}
$$

where

$$
\mathbf{e}_{l \omega}=\boldsymbol{\omega}_{i, l b}^{l b}-\boldsymbol{\omega}_{i, l d}^{l b}, \quad \mathbf{e}_{f \omega}=\boldsymbol{\omega}_{l b, f b}^{f b}-\omega_{l b, f d}^{f b} .
$$

We perform coordinate transformation of the attitude error such that $\mathbf{e}_{s q}=\left[1-h_{s} \tilde{\eta}_{s}, \tilde{\boldsymbol{\epsilon}}_{s}^{\top}\right]^{\top}$ which satisfies the kinematic equation

$$
\dot{\mathbf{e}}_{s q}=\mathbf{T}_{s e}^{\top}\left(\mathbf{e}_{s q}\right) \mathbf{e}_{s \omega}
$$

where

$$
\mathbf{T}_{s e}^{\top}\left(\mathbf{e}_{s q}\right)=\left[\begin{array}{c}
h_{s} \tilde{\boldsymbol{\epsilon}}_{s}^{\top} \\
\tilde{\eta}_{s} \mathbf{I}+\mathbf{S}\left(\tilde{\boldsymbol{\epsilon}}_{s}\right)
\end{array}\right],
$$

and the state variables $h_{s} \in H=\{-1,1\}$ determines the choice of goal equilibrium point.

\subsubsection{Control of leader}

The controller is given by

$$
\begin{aligned}
\boldsymbol{\tau}_{l a}^{l b} & =\mathbf{J}_{l} \dot{\boldsymbol{\omega}}_{l r}-\mathbf{S}\left(\mathbf{J}_{l} \boldsymbol{\omega}_{i, l b}^{l b}\right) \boldsymbol{\omega}_{l r}-k_{l q} \mathbf{T}_{l e}^{\top \mathbf{e}_{l q}} \mathbf{e}_{l q}-k_{l \omega} \mathbf{s}_{l}, \\
\boldsymbol{\omega}_{l r} & =\boldsymbol{\omega}_{i, l d}^{l b}-\gamma_{l} \mathbf{T}_{l e}^{\top}\left(\mathbf{e}_{l q}\right) \mathbf{e}_{l q}, \\
\mathbf{s}_{l} & =\boldsymbol{\omega}_{i, l b}^{l b}-\boldsymbol{\omega}_{l r}=\mathbf{e}_{l \omega}+\gamma_{l} \mathbf{T}_{l e}^{\top}\left(\mathbf{e}_{l q}\right) \mathbf{e}_{l q} .
\end{aligned}
$$

where $k_{l q}>0, k_{l \omega}>0$ and $\gamma_{l}>0$ are constant feedback gains. Next, let $\mathbf{x}_{2}=\left[\mathbf{e}_{l q}^{\top}, \mathbf{e}_{l \omega}^{\top}, h_{l}\right]^{\top}$, and for a given hysteresis margin $\sigma_{l}>0$ define the flow and jump sets, respectively as

$$
\begin{aligned}
& C_{l}=\left\{\left(\mathbf{e}_{l q}, \mathbf{e}_{l \omega}, h_{l}\right): h_{l}\left(k_{l q} \tilde{\eta}_{l}-\frac{1}{4} \gamma_{l} \tilde{\boldsymbol{\epsilon}}_{l}^{\top} \mathbf{J}_{l} \mathbf{e}_{l \omega}\right) \geq-\sigma_{l}\right\}, \\
& D_{l}=\left\{\left(\mathbf{e}_{l q}, \mathbf{e}_{l \omega}, h_{l}\right): h_{l}\left(k_{l q} \tilde{\eta}_{l}-\frac{1}{4} \gamma_{l} \tilde{\boldsymbol{\epsilon}}_{l}^{\top} \mathbf{J}_{l} \mathbf{e}_{l \omega}\right) \leq-\sigma_{l}\right\} .
\end{aligned}
$$

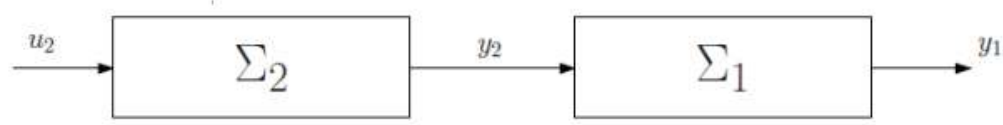

Fig. 2. Cascade interconnection of two dynamical systems. 
Then, the switching law is defined by

$$
\begin{array}{r}
\dot{h}_{l}=0 \quad \mathbf{x}_{2} \in C_{l} \\
\mathbf{x}_{2}^{+}=G_{l}\left(\mathbf{x}_{2}\right)=\left[\mathbf{e}_{l q}^{\top}, \mathbf{e}_{l \omega}^{\top},-h_{l}\right]^{\top} \mathbf{x}_{2} \in D_{l} .
\end{array}
$$

We have the following.

Proposition 1. Consider the system defined by (21) and (75)-(76) in closed-loop with the hybrid controller (77)-(79). Then, the set

$$
\mathcal{A}_{l}=\left\{\left(\mathbf{e}_{l q}, \mathbf{e}_{l \omega}, h_{l}\right):\left(\mathbf{e}_{l q}, \mathbf{e}_{l \omega}\right) \in \mathcal{B}_{\delta_{l}}\right\}
$$

where $\mathcal{B}_{\delta_{l}}=\left\{\mathbf{x}^{n} \in \mathbb{R}^{n}:\|\mathbf{x}\| \leq \delta_{l}\right\}$ and $\delta_{l}$ is to be defined, is Uniformly Asymptotically Stable (UAS) in the large on the set $\mathcal{S}^{3} \times \mathbb{R}^{3}$. That is, the equilibrium point $\left(\mathbf{e}_{l q}, \mathbf{e}_{l \omega}\right)=(\mathbf{0}, \mathbf{0})$ of the closed-loop system is Uniformly Practically Asymptotically Stable (UPAS) for all initial conditions in $\mathcal{S}^{3} \times \mathbb{R}^{3}$.

Proof: Define the LFC as

$$
V_{l}\left(\mathbf{x}_{2}\right)=\frac{1}{2}\left(\mathbf{s}_{l}^{\top} \mathbf{J}_{l} \mathbf{s}_{l}+\mathbf{e}_{l q}^{\top} k_{l q} \mathbf{e}_{l q}\right)>0 \quad \forall \mathbf{s}_{l} \neq \mathbf{0}, \mathbf{e}_{l q} \neq \mathbf{0} .
$$

Its total time derivative along the trajectories of the closed-loop system (21), (75)-(76) with the controller (77) and the switching law (79) yields

$$
\begin{aligned}
\dot{V}_{l}\left(\mathbf{x}_{2}\right)= & \mathbf{s}_{l}^{\top} \mathbf{S}\left(\mathbf{J}_{l} \boldsymbol{\omega}_{i, l b}^{l b}\right) \mathbf{s}_{l}-\mathbf{s}_{l}^{\top} k_{l \omega} \mathbf{s}_{l}-\mathbf{s}_{l}^{\top} k_{l q} \mathbf{T}_{l e}^{\top} \mathbf{e}_{l q} \\
& +\mathbf{e}_{l q}^{\top} k_{l q} \mathbf{T}_{l e} \mathbf{s}_{l}-\mathbf{e}_{l q}^{\top} k_{l q} \mathbf{T}_{l e} \gamma_{l} \mathbf{T}_{l e}^{\top} \mathbf{e}_{l q}+\mathbf{s}_{l}^{\top} \boldsymbol{\tau}_{l d}^{l b}
\end{aligned}
$$

and by inserting $(77 \mathrm{c})$ and applying the fact that $\mathbf{S}\left(\mathbf{J}_{l} \boldsymbol{\omega}_{i, l b}^{l b}\right)$ is skew-symmetric, we obtain

$$
\dot{V}_{l}=-\boldsymbol{\chi}_{2}^{\top} \mathbf{Q}_{l} \chi_{2}+\left(\mathbf{e}_{l \omega}+\gamma_{l} \mathbf{T}_{l e}^{\top} \mathbf{e}_{l q}\right)^{\top} \boldsymbol{\tau}_{l d}^{l b},
$$

where

$$
\mathbf{Q}_{l}=\left[\begin{array}{cc}
\gamma_{l} \mathbf{T}_{l e} \mathbf{T}_{l e}^{\top}\left(k_{l q}+\gamma_{l} k_{l \omega}\right) & \gamma_{l} k_{l \omega} \mathbf{T}_{l e} \\
\gamma_{l} k_{l \omega} \mathbf{T}_{l e}^{\top} & k_{l \omega} \mathbf{I}
\end{array}\right]=\left[\mathbf{q}_{i j}\right], \quad i, j=1,2,
$$

and $\boldsymbol{\chi}_{2}=\left[\mathbf{e}_{l q}^{\top}, \mathbf{e}_{l \omega}^{\top}\right]^{\top}$. According to (Horn \& Johnson, 1985) $\mathbf{Q}_{l}$ is positive definite if

$$
\begin{aligned}
\mathbf{q}_{22}=k_{l \omega} \mathbf{I} \succ \mathbf{q}_{21} \mathbf{q}_{11}^{-1} \mathbf{q}_{12} & =k_{l \omega}^{2} \gamma_{l}^{2} \mathbf{T}_{l e}^{\top}\left(\gamma_{l} \mathbf{T}_{l e} \mathbf{T}_{l e}^{\top}\left(k_{l q}+\gamma_{l} k_{l \omega}\right)\right)^{-1} \mathbf{T}_{l e} \\
& =\frac{k_{l \omega}^{2} \gamma_{l}}{k_{l q}+\gamma_{l} k_{l \omega}} \mathbf{I} \\
k_{l q}+\gamma_{l} k_{l \omega}>k_{l \omega} \gamma_{l} \rightarrow k_{l q}>0, &
\end{aligned}
$$

with the additional conservative condition that $k_{l \omega} \geq k_{l q}$, thus yielding

$$
\dot{V}_{l} \leq-q_{l m}\left\|\chi_{2}\right\|^{2}+\beta_{l d}\left(1+\frac{\gamma_{l}}{2}\right)\left\|\chi_{2}\right\|
$$

and $q_{l m}>0$ is the smallest eigenvalue of $\mathbf{Q}_{l}$. Thus $\dot{V}_{l}<0$ when $\left\|\boldsymbol{\chi}_{2}\right\|>\delta_{l}=\beta_{l d}\left(1+\gamma_{l} / 2\right) / q_{l m}$ and $\delta_{l}$ can be diminished by increasing $q_{l m}$ which is done by increasing the controller gains. The change in $V_{l}$ during jumps is expressed as

$$
V_{l}\left(G_{l}\left(\mathbf{x}_{2}\right)\right)-V_{l}\left(\mathbf{x}_{2}\right)=2 h_{l}\left(k_{l q} \tilde{\eta}_{l}-\frac{1}{4} \gamma_{l} \tilde{\boldsymbol{\epsilon}}_{l}^{\top} \mathbf{J}_{l} \mathbf{e}_{l \omega}\right)
$$


and by defining the flow and jump sets as in (78) we ensure that $V_{l}\left(G_{l}\left(\mathbf{x}_{2}\right)\right)-V_{l}\left(\mathbf{x}_{2}\right)<-2 \sigma_{l}$ when $\mathbf{x}_{2} \in D_{l}$, thus $V_{l}$ is strictly decreasing over jumps, and then

$$
\begin{aligned}
\dot{V}_{l}\left(\mathbf{x}_{2}\right) & \leq 0 \quad \forall \mathbf{x}_{2} \in C_{l} / \mathcal{B}_{\delta_{l}}, \\
V_{l}\left(G_{l}\left(\mathbf{x}_{2}\right)\right)-V_{l}\left(\mathbf{x}_{2}\right) & <0 \quad \forall \mathbf{x}_{2} \in D_{l} .
\end{aligned}
$$

Since the projection $\operatorname{Proj}_{\mathcal{S}^{3} \times \mathbb{R}^{3}} \mathcal{A}_{l}=\left\{\left(\mathbf{e}_{l q}, \mathbf{e}_{l \omega}, h_{l}\right):\left(\mathbf{e}_{l q}, \mathbf{e}_{l \omega}\right) \in \mathcal{B}_{\delta_{l}}\right\}$, according to (Sanfelice et al., 2007) renders the set $\mathcal{A}_{l}$ UAS with the basin of attraction $\mathcal{B}_{\mathcal{A}_{l}}=C_{l} \cup D_{l}$, thus UPAS in the large on the set $\mathcal{S}^{3} \times \mathbb{R}^{3}$ of the equilibrium point $\left(\mathbf{e}_{l q}, \mathbf{e}_{l \omega}\right)=(\mathbf{0}, \mathbf{0})$ of the closed-loop system follows.

Proposition 2. The interconnection term between the leader and follower spacecraft in (26), denoted as

is uniformly bounded.

$$
g(t, \mathbf{x})=-\mathbf{J}_{f} \mathbf{R}_{l b}^{f b} \mathbf{J}_{l}^{-1}\left(\boldsymbol{\tau}_{l d}^{l b}+\boldsymbol{\tau}_{l a}^{l b}\right)
$$

Proof: Since $V_{l}$ is positive definite and proper for $\left\|\chi_{2}\right\|>\delta_{l}$ we obtain that $\left\|\chi_{2}\right\|$ is bounded that is, for any $r>0$ there exists $\Delta(r)>0$ such that $\sup _{t>t_{0}}\left\|\chi_{2}(t)\right\| \leq \Delta$ for all initial conditions $\left\|\boldsymbol{\chi}_{2}\left(t_{0}\right)\right\|<r, t_{0} \geq 0$. Both $\left\|\boldsymbol{\omega}_{i, l d}^{l b}\right\| \leq \beta_{\boldsymbol{\omega}_{i, l d}^{l b}}$ and $\left\|\dot{\boldsymbol{\omega}}_{i, l d}^{l b}\right\| \leq \beta_{\dot{\boldsymbol{\omega}}_{i, l d}^{l b}}$, derived in Section 3 are continuous and bounded functions for some positive constants $\beta_{\omega_{i, l d}^{l l d}}^{b l}$ and $\beta_{\dot{\omega}_{i, l d}^{l b}}$. Thus it follows from (74) that $\left\|\omega_{i, l b}^{l b}\right\| \leq \beta_{\omega_{i, l b}^{l b}}$ is bounded by a positive constant $\beta_{\omega_{i, l b}^{l b}}$ for all $t \geq t_{0} \geq 0$. The derivative of $(77 \mathrm{~b})$ can be denoted as

$$
\dot{\boldsymbol{\omega}}_{l r}=\dot{\boldsymbol{\omega}}_{i, l d}^{l b}-\gamma_{l}\left(\dot{\mathbf{T}}_{l e}^{\top} \mathbf{e}_{l q}+\mathbf{T}_{l e}^{\top} \dot{\mathbf{e}}_{l q}\right)
$$

where

$$
\dot{\mathbf{T}}_{l e}^{\top} \mathbf{e}_{l q}=\frac{1}{2} \dot{\tilde{\boldsymbol{\epsilon}}}_{l}-\mathbf{T}_{l e}^{\top} \dot{\mathbf{e}}_{l q}=\left(\frac{1}{2}\left[\tilde{\eta}_{l} \mathbf{I}+\mathbf{S}\left(\frac{1}{2} \dot{\tilde{\boldsymbol{\epsilon}}}_{l}\right)\right]-\frac{1}{4}\right) \mathbf{e}_{l \omega},
$$

and by inserting (93) and (75) into (92) we obtain

$$
\dot{\boldsymbol{\omega}}_{l r}=\dot{\boldsymbol{\omega}}_{i, l d}^{l b}-\frac{\gamma_{l}}{2}\left[\tilde{\eta}_{l} \mathbf{I}+\mathbf{S}\left(\tilde{\boldsymbol{\epsilon}}_{l}\right)\right] \mathbf{e}_{l \omega} .
$$

Then by inserting (77b), (94) and (77c) into (77a) we obtain

$$
\begin{aligned}
\boldsymbol{\tau}_{l a}^{l b}= & \mathbf{J}_{l}\left(\dot{\boldsymbol{\omega}}_{i, l d}^{l b}-\frac{\gamma_{l}}{2}\left[\tilde{\eta}_{l} \mathbf{I}+\mathbf{S}\left(\tilde{\boldsymbol{\epsilon}}_{l}\right)\right] \mathbf{e}_{l \omega}\right) \\
& -\mathbf{S}\left(\mathbf{J}_{l} \boldsymbol{\omega}_{i, l b}^{l b}\right)\left[\boldsymbol{\omega}_{i, l d}^{l b}-\gamma_{l} \mathbf{T}_{l e}^{\top} \mathbf{e}_{l q}\right]-k_{l q} \mathbf{T}_{l e}^{\top} \mathbf{e}_{l q}-k_{l \omega}\left(\mathbf{e}_{l \omega}+\gamma_{l} \mathbf{T}_{l e}^{\top} \mathbf{e}_{l q}\right) .
\end{aligned}
$$

Note that $h_{l}$ is removed from the calculations since it won't have any impact on (96). Since all terms in (95) are either constant or upper bounded we have that $\left\|\boldsymbol{\tau}_{l a}^{l b}\right\| \leq \beta_{l a}$, where $\beta_{l a}$ is a positive constant, for all $t \geq t_{0} \geq 0$. The interconnection term (91) can thus be written as

$$
\|g(t, \mathbf{x})\| \leq j_{l M} j_{l m}\left(\beta_{l d}+\beta_{l a}\right),
$$

where $j_{l m} \leq\left\|\mathbf{J}_{l}\right\| \leq j_{l M}$, and we conclude that the interconnection term is uniformly bounded. 


\subsubsection{Control of leader-follower formation}

This section follows the line of Section 4.2.1 but we now talk of relative attitude and stress the point that the relative rotation and angular velocity is denoted $\mathbf{q}_{f}$ and $\boldsymbol{\omega}$, respectively. We define the control law as

$$
\begin{aligned}
\boldsymbol{\tau}_{f a}^{f b} & =\mathbf{J}_{f} \dot{\boldsymbol{\omega}}_{f r}+\mathbf{C}_{r}(\boldsymbol{\omega}) \boldsymbol{\omega}_{f r}+\mathbf{n}_{r}(\boldsymbol{\omega})-k_{f q} \mathbf{T}_{f e}^{\top}\left(\mathbf{e}_{f q}\right) \mathbf{e}_{f q}-k_{f \omega} \mathbf{s}_{f}, \\
\boldsymbol{\omega}_{f r} & =\boldsymbol{\omega}_{l b, f d}^{f b}-\gamma_{f} \mathbf{T}_{f e}^{\top}\left(\mathbf{e}_{f q}\right) \mathbf{e}_{f q} \\
\mathbf{s}_{f} & =\boldsymbol{\omega}_{l b, f b}^{f b}-\boldsymbol{\omega}_{f r}=\mathbf{e}_{f \omega}+\gamma_{f} \mathbf{T}_{f e}^{\top}\left(\mathbf{e}_{f q}\right) \mathbf{e}_{f q}
\end{aligned}
$$

where $k_{f q}>0, k_{f \omega}>0$ and $\gamma_{f}>0$ are constant gains. Next, let $\mathbf{x}_{1}=\left[\mathbf{e}_{f q}^{\top}, \mathbf{e}_{f \omega^{\prime}}^{\top}, h_{f}\right]^{\top}$ and, for a given hysteresis margin $\sigma_{f}>0$ define the flow and jump sets, respectively as

$$
\begin{aligned}
& C_{f}=\left\{\left(\mathbf{e}_{f q}, \mathbf{e}_{f \omega}, h_{f}\right): h_{f}\left(k_{f q} \tilde{\eta}_{f}-\frac{1}{4} \gamma_{f} \tilde{\boldsymbol{\epsilon}}_{f}^{\top} \mathbf{J}_{f} \mathbf{e}_{f \omega}\right) \geq-\sigma_{f}\right\}, \\
& D_{f}=\left\{\left(\mathbf{e}_{f q}, \mathbf{e}_{f \omega}, h_{f}\right): h_{f}\left(k_{f q} \tilde{\eta}_{f}-\frac{1}{4} \gamma_{f} \tilde{\boldsymbol{\epsilon}}_{f}^{\top} \mathbf{J}_{f} \mathbf{e}_{f \omega}\right) \leq-\sigma_{f}\right\} .
\end{aligned}
$$

Then, the switching law is defined by

$$
\begin{array}{r}
\dot{h}_{f}=0 \quad \mathbf{x}_{1} \in C_{f}, \\
\mathbf{x}_{1}^{+}=G_{f}\left(\mathbf{x}_{1}\right)=\left[\mathbf{e}_{f q^{\prime}}^{\top}, \mathbf{e}_{f \omega^{\prime}}^{\top}-h_{f}\right]^{\top} \mathbf{x}_{1} \in D_{f} .
\end{array}
$$

We have the following.

Proposition 3. Consider the system defined by (27) and (75)-(76) in closed-loop with the hybrid controller (97)-(99). Then, the set

$$
\mathcal{A}_{f}=\left\{\left(\mathbf{e}_{f q}, \mathbf{e}_{f \omega}, h_{f}\right):\left(\mathbf{e}_{f q}, \mathbf{e}_{f \omega}\right) \in \mathcal{B}_{\delta_{f}}\right\}
$$

where $\mathcal{B}_{\delta_{f}}=\left\{\mathbf{x}^{n} \in \mathbb{R}^{n}:\|\mathbf{x}\| \leq \delta_{f}\right\}$ and $\delta_{f}$ is to be defined, is UAS in the large on the set $\mathcal{S}^{3} \times \mathbb{R}^{3}$. That is, the equilibrium point $\left(\mathbf{e}_{f q}, \mathbf{e}_{f \omega}\right)=(\mathbf{0}, \mathbf{0})$ of the closed-loop system is UPAS for all initial conditions in $\mathcal{S}^{3} \times \mathbb{R}^{3}$.

Proof: Define the LFC as

$$
V_{f}\left(\mathbf{x}_{1}\right)=\frac{1}{2}\left(\mathbf{s}_{f}^{\top} \mathbf{J}_{f} \mathbf{s}_{f}+\mathbf{e}_{f q}^{\top} k_{f q} \mathbf{e}_{f q}\right)>0 \forall \mathbf{s}_{f} \neq \mathbf{0}, \mathbf{e}_{f q} \neq \mathbf{0} .
$$

Its total time derivative along the trajectories of the closed-loop system (27), (75)-(76) with the controller (97a)-(97c) and the switching law (99) yields

$$
\begin{aligned}
\dot{V}_{f}\left(\mathbf{x}_{1}\right)= & \mathbf{s}_{f}^{\top} \mathbf{C}_{r}(\boldsymbol{\omega}) \mathbf{s}_{f}-\mathbf{s}_{f}^{\top} k_{f \omega} \mathbf{s}_{f}-\mathbf{s}_{f}^{\top} k_{f q} \mathbf{T}_{f e}^{\top} \mathbf{e}_{f q}+\mathbf{e}_{f q}^{\top} k_{f q} \mathbf{T}_{f e} \mathbf{s}_{f} \\
& -\mathbf{e}_{f q}^{\top} k_{f q} \mathbf{T}_{f e} \gamma_{f} \mathbf{T}_{f e}^{\top} \mathbf{e}_{f q}+\mathbf{s}_{f}^{\top}\left[\boldsymbol{\tau}_{f d}^{f b}-\mathbf{J}_{l} \mathbf{R}_{l b}^{f b} \mathbf{J}_{l}^{-1}\left(\boldsymbol{\tau}_{l d}^{l b}+\boldsymbol{\tau}_{l a}^{l b}\right)\right]
\end{aligned}
$$

and by inserting (97c) and applying the fact that $\mathbf{C}(\boldsymbol{\omega})$ is skew-symmetric, we obtain

$$
\dot{V}_{f}\left(\mathbf{x}_{1}\right)=-\boldsymbol{\chi}_{1}^{\top} \mathbf{Q}_{f} \boldsymbol{\chi}_{1}+\left(\mathbf{e}_{f \omega}+\gamma_{f} \mathbf{T}_{f e}^{\top} \mathbf{e}_{f q}\right)^{\top}\left[\boldsymbol{\tau}_{f d}^{f b}-\mathbf{J}_{l} \mathbf{R}_{l b}^{f b} \mathbf{J}_{l}^{-1}\left(\boldsymbol{\tau}_{l d}^{l b}+\boldsymbol{\tau}_{l a}^{l b}\right)\right],
$$


where $\mathbf{Q}_{f}$ is similar to (84), and thus positive definite, and $\boldsymbol{\chi}_{1}=\left[\mathbf{e}_{l q}^{\top}, \mathbf{e}_{l \omega}^{\top}\right]^{\top}$. Eq. (103) can now be written as

$$
\dot{V}_{f}\left(\mathbf{x}_{1}\right) \leq-q_{f m}\left\|\chi_{1}\right\|^{2}+\beta_{f}\left\|\chi_{1}\right\|
$$

where $\beta_{f}=\beta_{f d}\left(1+\gamma_{f} / 2\right)+j_{l M} j_{l m}\left(\beta_{l d}+\beta_{l a}\right)$ and $q_{f m}>0$ is the smallest eigenvalue of $\mathbf{Q}_{f}$. Thus $\dot{V}_{f}<0$ when $\left\|\chi_{1}\right\|>\delta_{f}=\beta_{f} / q_{f m}$ and $\delta_{f}$ can be diminished by increasing $q_{f m}$ which is done by increasing the controller gains. The change in $V_{f}$ during jumps is expressed as

$$
V_{f}\left(G_{f}\left(\mathbf{x}_{1}\right)\right)-V_{f}\left(\mathbf{x}_{1}\right)=2 h_{f}\left(k_{f q} \tilde{\eta}_{f}-\frac{1}{4} \gamma_{f} \tilde{\boldsymbol{\epsilon}}_{f}^{\top} \mathbf{J}_{f} \mathbf{e}_{f \omega}\right),
$$

and by defining the flow and jump sets as in (98) we ensure that $V_{f}\left(G_{f}\left(\mathbf{x}_{1}\right)\right)-V_{f}\left(\mathbf{x}_{2}\right)<-2 \sigma_{f}$ when $\mathbf{x}_{1} \in D_{f}$, thus $V_{f}$ is strictly decreasing over jumps, and then

$$
\begin{aligned}
\dot{V}_{f}\left(\mathbf{x}_{1}\right) & \leq 0 \forall \mathbf{x}_{1} \in C_{f} / \mathcal{B}_{\delta_{f}}, \\
V_{f}\left(G_{f}\left(\mathbf{x}_{1}\right)\right)-V_{f}\left(\mathbf{x}_{1}\right) & <0 \forall \mathbf{x}_{1} \in D_{f} .
\end{aligned}
$$

Thus we conclude that the set $\mathcal{A}_{f}$ is UAS with the basin of attraction $\mathcal{B}_{\mathcal{A}_{f}}=C_{f} \cup D_{f}$, and UPAS in the large on the set $\mathcal{S}^{3} \times \mathbb{R}^{3}$ of the equilibrium points $\left(\mathbf{e}_{ \pm f q}, \mathbf{e}_{f \omega}\right)=(\mathbf{0}, \mathbf{0})$ of the closed-loop system follows.

It follows that since both the equilibrium point of the driving system and the equilibrium point of the driven system are UPAS in the large on the set $\mathcal{S}^{3} \times \mathbb{R}^{3}$ and the interconnection term is uniformly bounded, the equilibrium point of the closed-loop system of the total cascaded system on the form (69)-(70) is UPAS in the large on the set $\mathcal{S}^{3} \times \mathbb{R}^{3}$ (cf. Loría \& Panteley (2005)).

\section{Simulation results}

In this section we present simulation results where one leader and one follower spacecraft were tracking a common point on the Earth surface. The simulation was performed in Simulink using a fixed sample-time Runge-Kutta ODE4 solver, with sampling period equal to $0.1 \mathrm{~s}$. The leader spacecraft was flying in an elliptic Low Earth Orbit (LEO) with perigee at $600 \mathrm{~km}$, apogee at $750 \mathrm{~km}$, inclination $(i)$ at $79^{\circ}$ and the argument of perigee $(\omega)$ and the right ascension of the ascending node $(\Omega)$ at $0^{\circ}$. The tracking point was located on the Earth surface at zero degrees latitude and longitude, and both spacecraft continued tracking the point even if it was outside field of view. The spacecraft moments of inertia were $\mathbf{J}_{l}=\mathbf{J}_{f}=\operatorname{diag}\{4.350,4.337,3.664\} \mathrm{kgm}^{2}$ and spacecraft masses $m_{l}=m_{f}=100 \mathrm{~kg}$. The initial conditions were set to $\mathbf{p}\left(t_{0}\right)=[0,-100,0]^{\top} \mathrm{m}, \dot{\mathbf{p}}\left(t_{0}\right)=\ddot{\mathbf{p}}\left(t_{0}\right)=\mathbf{0}$, $\mathbf{q}_{l}\left(t_{0}\right)=\mathbf{q}_{f}\left(t_{0}\right)=[0.9437,0.1277,0.1449,-0.2685]^{\top}, \boldsymbol{\omega}_{i, l b}^{l b}\left(t_{0}\right)=\left[\begin{array}{lll}1.745 & -3.491 & 0.873\end{array}\right]^{\top} \times$ $10^{-3} \mathrm{rad} / \mathrm{s}$ and $\omega_{i, f b}^{f b}\left(t_{0}\right)=[0,0,0]^{\top} \mathrm{rad} / \mathrm{s}$. The desired conditions for relative translation were set to $\mathbf{p}_{d}=[0,-1000,500]^{\top} \mathrm{m}, \dot{\mathbf{p}}_{d}=\ddot{\mathbf{p}}_{d}=\mathbf{0}$, while the desired values for relative rotation were calculated based on the results presented in Section 3. The controller gains were set to $\mathbf{K}_{p}=\mathbf{K}_{d}=\{0.5\} \mathbf{I}$ for control of relative translation, and $k_{l q}=k_{f q}=5, k_{l \omega}=k_{f \omega}=10$ and $\gamma_{l}=\gamma_{f}=1$ for control of relative rotation.

Since we are considering a slightly elliptic LEO, we only consider the disturbance torques which are the major contributors to these kind of orbits; namely, gravity gradient torque (Sidi, 1997), and forces and torques caused by atmospheric drag (Wertz, 1978) and $J_{2}$ effect (Montenbruck \& Gill, 2001). The $J_{2}$ effect is caused by non-homogeneous mass distribution 

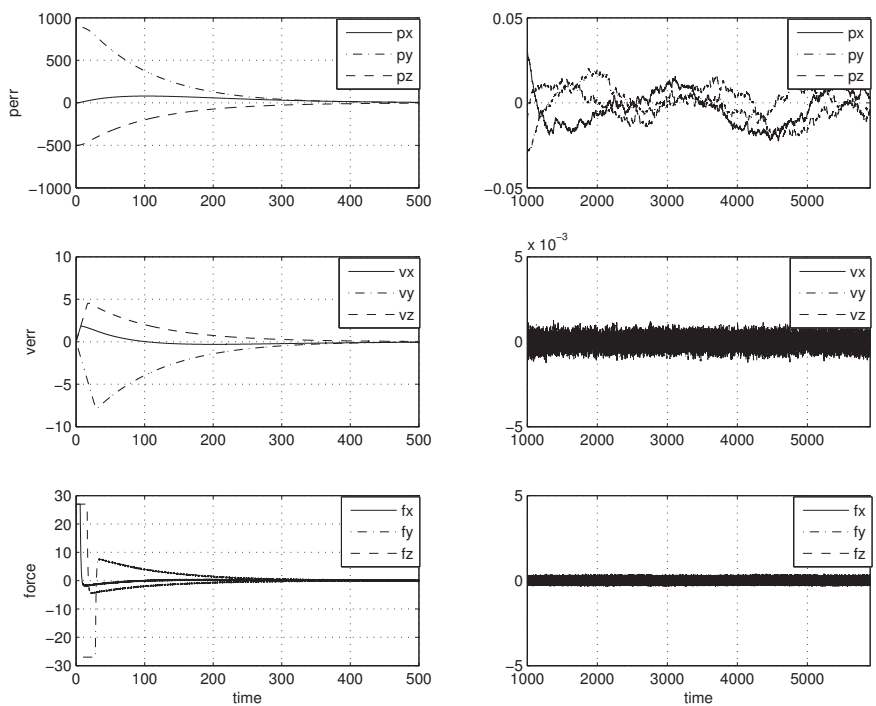

Fig. 3. Relative position error, relative velocity error and control force with uncompensated disturbances and unfiltered sensor noise.
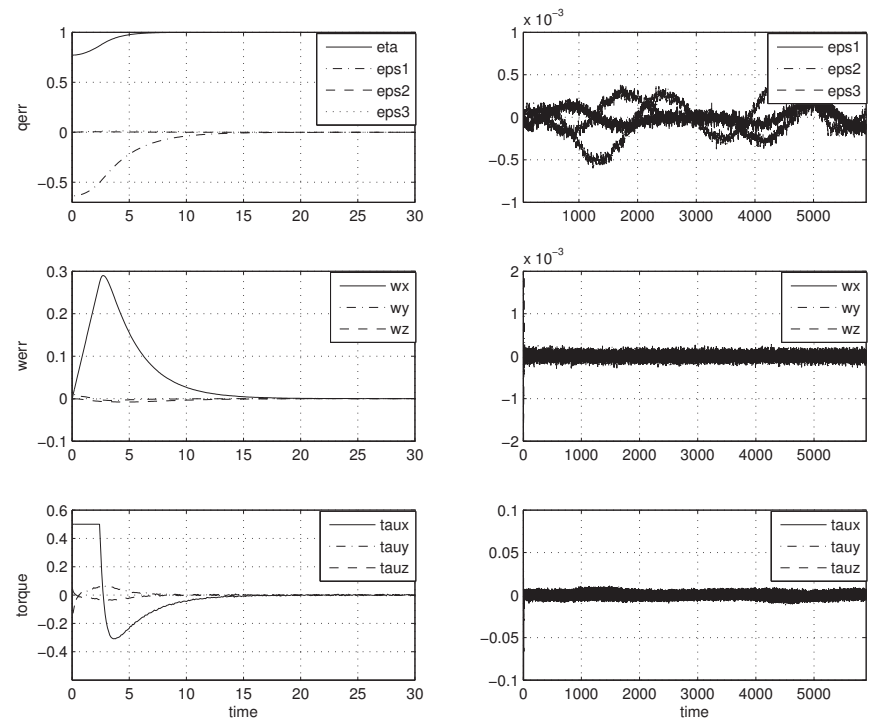

Fig. 4. Relative attitude error, relative angular velocity error and control torque with uncompensated disturbances and unfiltered sensor noise. 
of a planet. The torques generated by atmospheric drag and $J_{2}$ are induced because of a $10 \mathrm{~cm}$ displacement of the center of mass. All disturbances were considered continuous and bounded. The noise was considered to be contained in a compact $\sigma \mathbb{B}^{n}=\left\{x \in \mathbb{R}^{n}:\|x\| \leq \sigma\right\}$ and a suitable amount were added to the measured vectors such that $\mathbf{r}_{l n}=\mathbf{r}_{l}+0.01 \mathbb{B}^{3}$, $\mathbf{p}_{n}=\mathbf{p}+0.01 \mathbb{B}^{3}, \mathbf{p}_{l n}=\mathbf{p}_{l}+5 \times 10^{-3} \mathbb{B}^{3}, \dot{\mathbf{p}}_{n}=\dot{\mathbf{p}}+5 \times 10^{-3} \mathbb{B}^{3}$. The measured states $\mathbf{q}_{l n}$ and $\mathbf{q}_{n}$ satisfies $\mathbf{q}_{l n} \in\left(\mathbf{q}_{l}+\left[0,\left(0.001 \mathbb{B}^{3}\right)^{\top}\right]^{\top}\right) \cap \mathcal{S}^{3}$ and $\mathbf{q}_{n} \in\left(\mathbf{q}+\left[0,\left(0.001 \mathbb{B}^{3}\right)^{\top}\right]^{\top}\right) \cap \mathcal{S}^{3}$, and $\boldsymbol{\omega}_{l n}=\boldsymbol{\omega}_{i, l b}^{l b}+2 \times 10^{-3} \mathbb{B}^{3}$ and $\boldsymbol{\omega}_{n}=\boldsymbol{\omega}+2 \times 10^{-3} \mathbb{B}^{3}$. The simulation time is set to one orbital period (5896 s) to show the performance of the reference generation scheme.
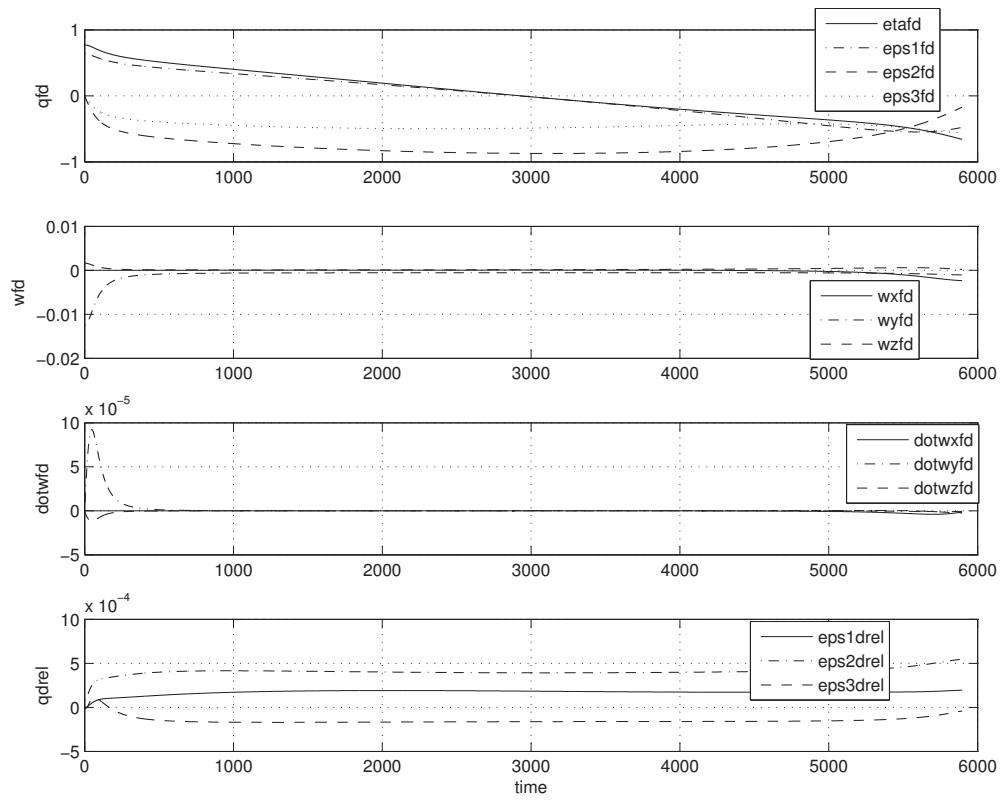

Fig. 5. Follower desired attitude, angular velocity, angular acceleration and relative desired attitude with uncompensated disturbances and unfiltered sensor noise.

Figure 3 shows the relative position, velocity and control force during both settling and station keeping phase. As can be seen the position of the follower spacecraft converged toward the desired position and was then stationed there within a few centimeters during the orbital period. Figure 4 shows the relative attitude, angular velocity and control torque during settling and station keeping phase, and results similar to the relative translation can be observed for relative rotation as well. It can further be shown that the fluctuations caused by the disturbances can be diminished by increasing the controller gains. The states are converging towards the equilibrium point and kept close during the following orbit. The three topmost plots in Figure 5 shows the desired attitude, angular velocity and angular acceleration for the leader spacecraft during one orbit. What can be seen is that there is no rotation about the $x$-axis during the orbital period. It should also be mentioned that the desired angular velocity and acceleration are smaller during the second pass compared to the 
first. This is because the Earth is rotating, thus the distance between the spacecraft and target is longer, causing less fluctuation on the components. The bottommost plot in Figure 5 shows the epsilon components of the difference in attitude between desired leader and follower, defined as $\tilde{\boldsymbol{\epsilon}}_{d}=\eta_{l d} \boldsymbol{\epsilon}_{f d}-\eta_{f d} \boldsymbol{\epsilon}_{l d}-\mathbf{S}\left(\boldsymbol{\epsilon}_{f d}\right) \boldsymbol{\epsilon}_{l d}$. It can be seen that since the follower spacecraft was moving away from the leader spacecraft during the first 500 seconds, the difference in desired relative attitude increased during the same period.
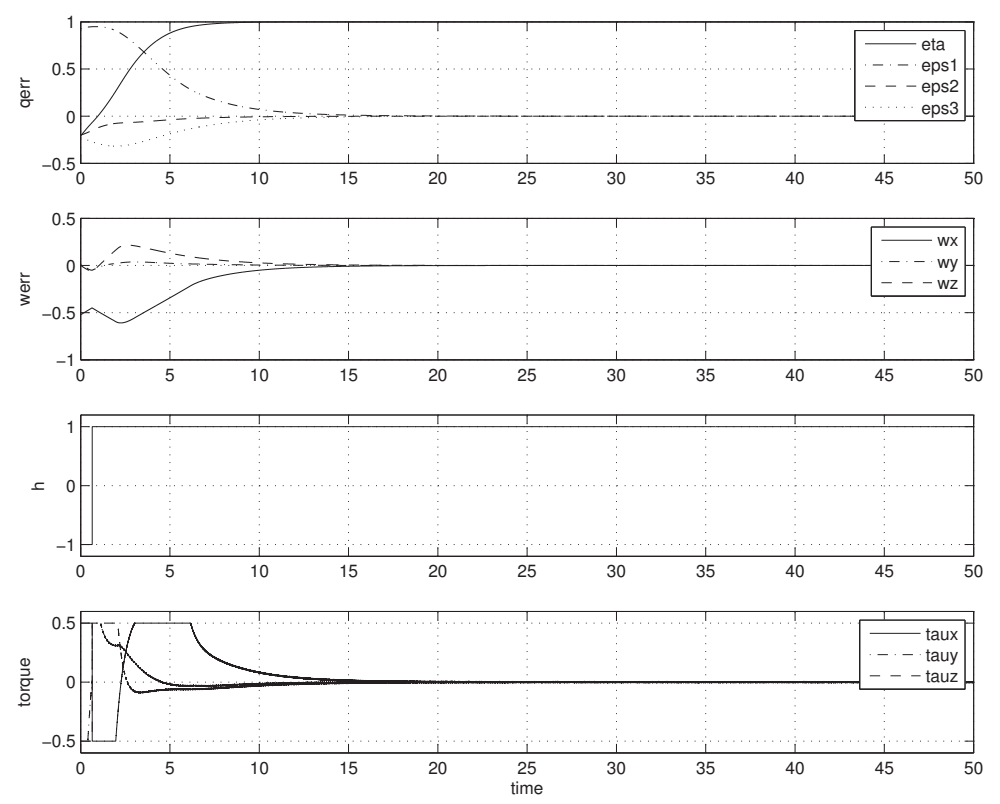

Fig. 6. Relative attitude error, relative angular velocity error, switching value and control torque with uncompensated disturbances and unfiltered sensor noise.

Figure 6 shows simulation results where the follower spacecraft starts with an initial attitude of $\mathbf{q}_{f}\left(t_{0}\right)=[-0.866,0.5,0,0]^{\top}$ and $\omega_{i, f b}^{f b}\left(t_{0}\right)=[-0.5236,0,0]^{\top} \mathrm{rad} / \mathrm{s}$. As can be seen, the negative equilibrium point is the closest one, thus $h_{f}=-1$, but a switch occurs because of the initial angular velocity, driving the states towards the positive equilibrium.

\section{Conclusions}

In this paper we have presented a scheme for calculating the desired attitude, angular velocity and angular acceleration for a leader spacecraft and relative attitude, angular velocity and angular acceleration for a leader-follower spacecraft formation. Sliding surface tracking control laws were presented for both relative translational and rotational control of a leader and follower in cascades. For relative position the equilibrium point of the closed-loop system was proven globally uniformly asymptotically stable, while for relative rotation, the equilibrium point of the cascaded closed-loop systems was proven uniformly practically asymptotically stable in the large on the set $\mathcal{S}^{3} \times \mathbb{R}^{3}$, both control laws were considered 
to be perturbed by unknown but bounded disturbances which were not compensated for. Simulation results were presented to show both the performance of the control laws, and that the presented scheme enables a leader-follower formation to perform measurements on a common point on the Earth surface even during formation reconfiguration.

\section{References}

Battin, R. H. (1999). An Introduction to the Mathematics and Methods of Astrodynamics, Revised Edition, AIAA Education Series, American Institute of Aeronautics and Astronautics, Reston, VA. ISBN 1-56347-342-9.

Berghuis, H. \& Nijmeijer, H. (1993). A passivity approach to controller-observer design for robots, IEEE Transactions on Robotics and Automation 9(6): 740-754.

Casagrande, D., A. A. P. T. (2008). Global asymptotic stabilization of the attitude and the angular rates of an underactuated non-symmetric rigid body, Automatica 44: 1781-1789.

Chaillet, A. (2006). On Stability and Robustness of Nonlinear Systems: Applications to Cascaded Systems, PhD thesis, UFR Scientifique D'Orsay, Paris, France.

Chaillet, A. \& Loría, A. (2008). Uinform semiglobal practical asymptotic stability for non-autonomous cascaded systems and applications, Automatica 44: 337-347.

Chen, X., Steyn, W. H. \& Hashida, Y. (2000). Ground-target tracking control of earth-pointing satellites, AIAA Guidance, Navigation, and Control Conference and Exhibit, Denver, CO, pp. 1834-1844.

Clohessy, W. H. \& Wiltshire, R. S. (1960). Terminal guidance system for satellite rendezvous, Journal of Aerospace Sciences 27(9): 653-658.

Egeland, O. \& Gravdahl, J. T. (2002). Modeling and Simulation for Automatic Control, Marine Cybernetics, Trondheim, Norway. ISBN 82-92356-01-0.

Fossen, T. I. \& Fjellstad, O.-E. (1993). Cascaded adaptive control of ocean vehicles with significant actuator dynamics, Proceedings of the IFAC World Congress, Sydney, Australia.

Garcia, I. \& How, J. P. (2005). Trajectorie optimization for satellite reconfiguration maneuvers with position and attitude constraints, Proceedings of the American Control Conference, Portland, OR.

Goebel, R., Sanfelice, R. G. \& Teel, A. R. (2009). Hybrid dynamical systems, IEEE Control Systems Magazine 29(2): 28-93.

Goerre, B. B. \& Shucker, B. (1999). Geometric attitude control of a small satellite for ground tracking maneuvers, Proceedings of the 13th AIAA/USU Conference on Small Satellites, Utah State University.

Grøtli, E. I. (2010). Robust stability and control of spacecraft formations, PhD thesis, Norwegian University of Science and Technology.

Hahn, W. (1967). Stability of Motion, Springer-Verlag, Berlin, Germany.

Hill, G. W. (1878). Researches in the lunar theory, American Journal of Mathematics 1(1): 5-26.

Horn, R. A. \& Johnson, C. R. (1985). Matrix Analysis, Cambridge University Press. ISBN 0-521-38632-2.

Janković, M., Sepulchre, R. \& Kokotović, P. V. (1996). Constructive lyapunov stabilization of non linear cascaded systems, IEEE Transactions on Automatic Control 41: 1723-1736.

Jiang, Z. P. \& Mareels, I. (1997). A small gain control method for nonlinear cascaded systems with dynamic uncertainties, IEEE Transaction on Automatic Control 42: 1-17.

Kristiansen, R. (2008). Dynamic Synchronization of Spacecraft - Modeling and Coordinated Control 
of Leader-Follower Spacecraft Formations., PhD thesis, Department of Engineering Cybernetics, Norwegian University of Science and Technology, Trondheim, Norway. Liberzon, D. (2003). Switching in Systems and Control, Birkhäuser. ISBN 0-8176-4297-8.

Loría, A., Nijmeijer, H. \& O., E. (1998). Cascaded synchronization of two pendula, Proceedings of the American Control Conference.

Loría, A. \& Panteley, E. (2005). Cascaded nonliear time-varying systems: Analysis and design, Advanced Topics in Control Systems Theory, Vol. 311 of Lecture Notes in Control and Information Sciences, Springer Verlag, chapter 2, pp. 23-64. ISBN 1-85233-923-3.

Mayhew, C. G., Sanfelice, R. G. \& Teel, A. R. (2009). Robust global asymptotic attitude stabilization of a rigid body by quaternion-based hybrid feedback, Proceedings of the IEEE Conference on Decision and Control.

McInnes, C. R. (1995). Autonomous ring formation for a planar constellation of satellites, AIAA Journal of Guidance, Control and Dynamics 18(5): 1215-1217.

Montenbruck, O. \& Gill, E. (2001). Satellite Orbits. Models, methods, applications, Springer-Verlag, Berlin, Germany. First edition, corrected second printing, ISBN 3-540-67280-X.

Paden, B. \& Panja, R. (1988). Globally asymptotically stable 'PD+' controller for robot manipulators, International Journal of Control 47(6): 1697-1712.

Panteley, E. \& Loría, A. (1998). On global uniform asymptotic stability of nonlinear time-varying systems in cascade, Systems \& Control Letters 33(2): 131-138.

Sanfelice, R. G., Goebel, R. \& Teel, A. R. (2007). Invariance principles for hybrid systems with connections to detectability and asymptotic stability, IEEE Transactions on Automatic Control 52(12): 2282-2297.

Sidi, M. J. (1997). Spacecraft Dynamics and Control, Cambridge University Press, New York. ISBN 0-521-78780-7.

Slotine, J. J.-E. \& Li, W. (1987). On the adaptive control of robot manipulators, International Journal of Robotics Research 6: 49-59.

Steyn, W. H. (2006). A view finder control system for an earth observation satellite, Aerospace Science and Technology 10(3): 248-255.

Takegaki, M. \& Arimoto, S. (1981). A new feedback method for dynamic control of manipulators, ASME Journal of Dynamical Systems, Measurement and Control 102: 119-125.

Tsiotras, P., Shen, H. \& Hall, C. (2001). Satellite attitude control and power tracking with energy/momentum wheels, AIAA Journal of Guidance, Control and Dynamics 24(1): 23-34.

Wang, P. K. C. \& Hadaegh, F. Y. (1996). Coordination and control of multiple microspacecraft moving in formation, Journal of the Astronautical Sciences 44(3): 315-355.

Wertz, J. R. (ed.) (1978). Spacecraft Attitude Determination and Control, Kluwer Academic Publishers, London. ISBN 90-277-0959-9.

Wong, H. \& Kapila, V. (2005). Spacecraft formation flying near sun-earth $l_{2}$ lagrange point: Trajectory generation and adaptive output feedback control, Proceedings of the American Control Conference, Portland, OR.

Yan, H., Alfriend, K. T., Vadali, S. R. \& Sengupta, P. (2009). Optimal design of satellite formation relative motion orbits using least-square methods, AIAA Journal of Guidance, Control and Dynamics 32(2): 599-604.

Yan, Q., Yang, G., Kapila, V. \& de Queiroz, M. (2000a). Nonlinear dynamics and adaptive control of multiple spacecraft in periodic relative orbits, Proceedings of the AAS 
Guidance and Control Conference, Breckenridge, CO.

Yan, Q., Yang, G., Kapila, V. \& de Queiroz, M. (2000b). Nonlinear dynamics and output feedback control of multiple spacecraft in elliptical orbits, Proceedings of the American Control Conference, Chicago, Illinois. 


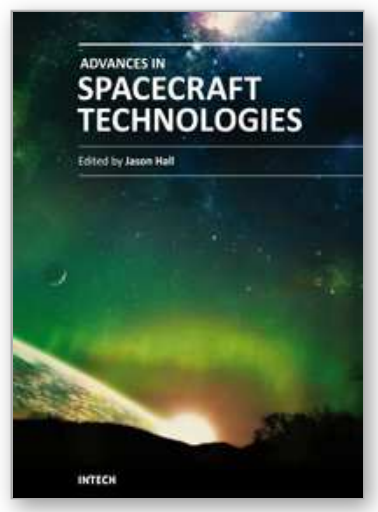

\author{
Advances in Spacecraft Technologies \\ Edited by Dr Jason Hall
}

ISBN 978-953-307-551-8

Hard cover, 596 pages

Publisher InTech

Published online 14, February, 2011

Published in print edition February, 2011

The development and launch of the first artificial satellite Sputnik more than five decades ago propelled both the scientific and engineering communities to new heights as they worked together to develop novel solutions to the challenges of spacecraft system design. This symbiotic relationship has brought significant technological advances that have enabled the design of systems that can withstand the rigors of space while providing valuable space-based services. With its 26 chapters divided into three sections, this book brings together critical contributions from renowned international researchers to provide an outstanding survey of recent advances in spacecraft technologies. The first section includes nine chapters that focus on innovative hardware technologies while the next section is comprised of seven chapters that center on cutting-edge state estimation techniques. The final section contains eleven chapters that present a series of novel control methods for spacecraft orbit and attitude control.

\title{
How to reference
}

In order to correctly reference this scholarly work, feel free to copy and paste the following:

Rune Schlanbusch and Per Johan Nicklasson (2011). Synchronization of Target Tracking Cascaded LeaderFollower Spacecraft Formation, Advances in Spacecraft Technologies, Dr Jason Hall (Ed.), ISBN: 978-953307-551-8, InTech, Available from: http://www.intechopen.com/books/advances-in-spacecrafttechnologies/synchronization-of-target-tracking-cascaded-leader-follower-spacecraft-formation

\section{INTECH}

open science | open minds

\section{InTech Europe}

University Campus STeP Ri

Slavka Krautzeka 83/A

51000 Rijeka, Croatia

Phone: +385 (51) 770447

Fax: +385 (51) 686166

www.intechopen.com

\section{InTech China}

Unit 405, Office Block, Hotel Equatorial Shanghai

No.65, Yan An Road (West), Shanghai, 200040, China 中国上海市延安西路65号上海国际贵都大饭店办公楼 405 单元

Phone: +86-21-62489820

Fax: $+86-21-62489821$ 
(C) 2011 The Author(s). Licensee IntechOpen. This chapter is distributed under the terms of the Creative Commons Attribution-NonCommercialShareAlike-3.0 License, which permits use, distribution and reproduction for non-commercial purposes, provided the original is properly cited and derivative works building on this content are distributed under the same license. 